\title{
Improved high gain observer design for a class of disturbed nonlinear systems
}

\author{
M. Farza • A. Ragoubi - S. Hadj Saïd • \\ M. M'Saad
}

Received: 3 February 2021 / Accepted: 26 August 2021 / Published online: 9 September 2021

(C) The Author(s) 2021

\begin{abstract}
This paper provides a redesigned version of the Standard High Gain Observer (SHGO) to cope with the peaking phenomenon occurring during the transient periods as well as the sensitivity to high frequency measurement noise. The observer design is performed for a class of uniformly observable systems with noise free as well as noisy output measurements and the resulting observer is referred to as Non Peaking Filtered High Gain Observer (NPFHGO). The NPFHGO shares the same structure as its underlying SHGO and differs only by its corrective term which is still parameterized by a unique positive scalar up to an appropriate expression involving nested saturations. Of a fundamental interest, the power of the scalar parameter does not exceed one unlike in the case of the SHGO where this power grows from 1 to the system dimension. Moreover, it is shown that the equations of the NPFHGO become identical to those of the SHGO after a transient time horizon that can made arbitrarily small for sufficiently high values of the design parameter. A particular emphasis is put on the case of systems with noisy output measurements. It is shown how a multiple integrator of the corrupted outputs can be cascaded with the original system leading to an augmented system included in the class of
\end{abstract}

M. Farza $(\varangle) \cdot$ A. Ragoubi $\cdot$ M. M'Saad

UNICAEN, ENSICAEN, LAC, Normandie University,

14000 Caen, France

e-mail: mondher.farza@unicaen.fr

A. Ragoubi · S. Hadj Saïd

National School of Engineering of Monastir,

LA2SE, University of Monastir, Monastir, Tunisia systems for which the NPFHGO has been designed. The performance and main properties of the NPFHGO are highlighted and compared to those of its underlying SHGO through simulation results involving a single link robot arm system.

Keywords Nonlinear systems - Standard high gain observers $\cdot$ Peaking phenomenon - Saturation functions · Filtered high gain observers · Descartes' rule of signs

\section{Mathematics Subject Classification Systems theory $\cdot$ Control}

\section{Introduction}

An intensive research activity has been devoted to the observer design for uniformly observable systems over the last four decades (see for instance [1-10] and references therein). A particular emphasis has been put on the Standard High Gain Observer (SHGO) design for fundamental as well as simplicity purposes [5]. Indeed, SHGO have been extensively used for the output feedback control design of nonlinear systems (see [11] and references therein). The structure of a SHGO is very simple since it consists in a copy of the system dynamics up to a corrective term given by the product of the observer gain by the output observation error. There are two features of the SHGO that are worth to be mentioned. Firstly, the observer gain is param- 
eterized by a unique design parameter, i.e. a positive scalar denoted $\theta$, which has to be taken generally high enough to ensure the underlying observation error convergence. Secondly, the design parameter $\theta$ intervenes with positive powers ranged from one to the system dimension, commonly denoted $n$. These features could be essentially crucial from numerical implementation point of views for systems with high dimensions when relatively high values of $\theta$ are required. Moreover, there are two main issues of SHGO that have to be pointed out, namely the peaking of the observer state variables during the transient periods and the observer sensitivity to the output measurement noise.

Several solutions have been recently proposed to alleviate the sensitivity to measurement noise of SHGO [12-17]. A redesigned version of SHGO has been proposed in [15] for a class of nonlinear systems of dimension $n$. Though the observer dimension is equal to $2(n-1)$, the underlying observer gain is parameterized by a scalar design parameter the power of which is limited to 2. Such a design feature allows to enhance the observer performance with respect to the ubiquitous measurement noise. The same objective has bee investigated in [14] using a cascade observer the dimension of which is equal to $2 n$. More specifically, the observer consists in cascading two subsystems of the same dimension as the original system. The first subsystem is nothing than a copy of the original system with a simple correction term which linearly depends on the state of the second subsystem, while the second subsystem is a linear filter driven by the output observation error, namely the error between the output measurement and an output prediction provided by the first subsystem.

The peaking phenomenon is a challenging problem that has been recently investigated [18-22]. A suitable redesign of the SHGO has been proposed in [19] leading to an observer of the same dimension as the original system where the power of the observer gain is limited to one. The observer design proposed in [15] has been reconsidered in [20] to deal with the peaking phenomenon by properly using nested saturation functions.

In the present paper, one aims at investigating the design of an observer with filtering capabilities while allowing a significant reduction of the peaking phenomenon. Such observer shall be referred to as Non Peaking Filtering High Gain Observer (NPFHGO) and its design shall be achieved in a context accounting for the free noise output case and the noisy output case as well. The unifying of both cases is made possible by considering a class of uniformly observable systems where the expression of the outputs only depends on the system state. Such outputs may coincide with the system real outputs if the latter are assumed to be noise free. In the case where the real measurements are noisy, the system outputs correspond to a filtered version of the real measurement outputs, i.e they are the outputs of an appropriate filter, namely a multiple integrator, which is a part of the system and the entry of which are the real measurement outputs. Moreover, two unknown essentially bounded functions are involved in the class of systems. The first one is treated as a disturbance while the second one allows to identify the case where the class of considered system involves a filter that provides the system outputs.

The main motivation behind the consideration of the above class of systems is to move away in a first step the problem dealing with the observer sensitivity to noise and to focus only on the problem dealing with the peaking phenomenon. Indeed, a Non Peaking High Gain Observer (NPHGO), the structure of which is very similar to that of its underlying SHGO, is designed for the above class system. The main difference between both observers lies in the corrective term of the NPHGO which is expressed through nested saturation functions. More specifically, the corrective term is parameterized by a scalar design parameter $\theta$ the power of which does not exceed one. Two fundamental properties of the NPHGO have to be emphasized. The first property deals with the fact that under an appropriate assumption on the essential bound of the second unknown function, it is shown that the state estimate is bounded during the transient periods by a function $\alpha(\theta)$ such that $\lim _{\theta \rightarrow \infty}(\alpha(\theta) / \theta)=0$. The second property consists in the fact that the NPHGO equations become identical to those of its underlying SHGO after a transient time horizon that can be made arbitrarily small by choosing sufficiently high values of the design parameter $\theta$.

It is worth noticing that the NPHGO becomes a NPFHGO when it is designed for systems with filtered outputs. Such an issue shall be fully detailed by considering a class of systems with noisy outputs which shall be cascaded with an appropriate multiple integrator of the outputs in such a way that the augmented system is included in the class of systems for which a NPHGO can be designed. Then one shall show how the 
so designed NPHGO is specialized and performs as a NPFHGO.

The paper is organized as follows. The problem formulation is presented in Sect. 2 with a particular emphasis on the considered class of systems and the SHGO design assumptions. The equations of the SHGO are briefly presented and its main properties, established in [23], are recalled. The design of the NPHGO is detailed in Sect. 3 with a particular emphasis on the appropriate nested saturation functions involved in the observer gain. The main steps of the observer design are put forward through five propositions and the main properties of the proposed NPHGO resulting from these propositions are summarized in a theorem. In Sect. 4, the design of a NPFHGO for a class of systems with noisy outputs is emphasized. It is shown how a system with noisy outputs can be augmented by a multiple integrator of these noisy outputs in such a way that the augmented system is included in the class of systems for which a NPHGO can be designed. Then, the equations of the underlying NPHGO are derived and it is shown how this observer acts as a NPFGHO, namely it significantly reduces the peaking of the state variables during the transient periods while improving the sensitivity to noise of the observer. In Sect. 5, the performance of the proposed NPFHGO and its main properties are highlighted and compared to those of a SHGO through an example dealing with a single-link robot arm system. Finally, concluding remarks are given in Sect. 6. For clarity purposes, the proofs of all propositions are detailed in Appendices A-E.

Throughout the paper, $I_{k}$ denotes the $k \times k$ identity matrix; $\|\cdot\|$ denotes the euclidian norm; for any Symmetric Positive Definite (SPD) matrix $Q, \lambda_{Q}^{(M)}$ (resp. $\left.\lambda_{Q}^{(m)}\right)$ denotes its maximum (resp. minimum) eigenvalue and $\sigma_{Q}=\sqrt{\lambda_{Q}^{(M)} / \lambda_{Q}^{(m)}}$ is its conditioning number. More specifically, one defines the following matrices.

$$
\begin{aligned}
& A_{1}=0, \quad A_{k}=\left(\begin{array}{cc}
0 & I_{k-1} \\
0 & 0
\end{array}\right) \in R^{k \times k} \text { for } k \geq 2, \\
& C_{k}=\left(\begin{array}{lll}
1 & 0 \ldots 0
\end{array}\right) \in \mathbb{R}^{k} \text { for } k \geq 1, \\
& B_{k, k_{0}} \in \mathbb{R}^{k}, \quad k \geq 2, \quad 1 \leq k_{0} \leq k \text { with } \\
& B_{k, k_{0}}\left(k_{0}\right)=1 \text { and } B_{k, k_{0}}(i)=0, \\
& \quad i \in\{1, \ldots, k\} \backslash\left\{k_{0}\right\},
\end{aligned}
$$

$$
\begin{aligned}
& \Gamma_{k}=\left(\gamma_{1} \ldots \gamma_{k}\right)^{T}, \quad \bar{A}_{k} \triangleq A_{k}-\Gamma_{k} C_{k} \text { for } k \\
& \quad \geq 1, \\
& U_{k}=(1 \ldots 1)^{T} \in \mathbb{R}^{k} \text { for } k \geq 1, \\
& \Delta_{k}(\theta)=\operatorname{diag}\left(1, \frac{1}{\theta}, \ldots, \frac{1}{\theta^{k-1}}\right) \text { for } k \geq 1 .
\end{aligned}
$$

The parameter $\theta$ involved in the expression of $\Delta_{k}(\theta)$ is a real positive number.

On other aspects, let $n \geq 1$ be a positive integer and let $\xi^{T}=\left(\xi_{1} \ldots \xi_{n}\right) \in \mathbb{R}^{n}$ with $\xi_{i} \in \mathbb{R}, i=1, \ldots, n$; then for any integer $1 \leq k \leq n$, one defines $\underline{\xi}_{k} \in \mathbb{R}^{k}$ as follows

$\underline{\xi}_{k}^{T}=\left(\xi_{1} \ldots \xi_{k}\right) \in \mathbb{R}^{k}, \quad k=1, \ldots, n$.

Finally, one recalls a useful property: If $\mathcal{A}_{k}$ is a $n \times n$ Hurwitz matrix, then there exist a $k \times k$ SPD matrix $P_{k}$ and a positive real $\mu_{k}$ such that

$P_{k} \mathcal{A}_{k}+\mathcal{A}_{k}^{T} P_{k} \leq-2 \mu_{k} I_{k}$.

\section{The problem formulation}

One aims at providing a NPFHGO for a class of disturbed nonlinear systems described by the following equations

$$
\left\{\begin{array}{l}
\dot{x}=A_{n} x+\varphi(u, x)+B_{n, n} \varepsilon(t) \\
y=C_{n} x+v(t)=x_{1}+v(t)
\end{array}\right.
$$

where $x=\left(x_{1} \ldots x_{n}\right)^{T} \in \mathbb{R}^{n}$ denotes the state of the system with $x_{i} \in \mathbb{R}$ for $i=1, \ldots, n ; u \in \mathbb{R}$ denotes the system input and $y \in \mathbb{R}$ denotes the output of the system, the matrices $A_{n}, C_{n}$ and $B_{n, n}$ are defined as in (1)-(3) with $k=k_{0}=n, v(t)$ is the output noise and the function $\varepsilon:[0,+\infty[\mapsto \mathbb{R}$ denotes the system uncertainties and may depend on the input and uncertain parameters. It shall be treated as an unknown function which explicitly depends on time $t$ for $t \geq 0$. Finally, $\varphi(u, x) \in \mathbb{R}^{n}$ denotes a nonlinear vector field and each of its $n$ components has a triangular structure with respect to $x$, i.e. $\varphi_{i}(u, x)=\varphi_{i}\left(u, \underline{x}_{i}\right)$ for $i=1, \ldots, n$ where $\underline{x}_{i}$ is the sub-vector of $x$ as defined by (7).

The class of systems (9) is included in a class of systems considered in [23] for which a SHGO has been designed under appropriate assumptions usually used in the context of high gain observer design. As stated above, the main motivation behind the NPFHGO design is to cope with the peaking phenomenon occurring during the transient periods and to improve the 
sensitivity of the observer with respect to output measurements noise. For this end, one shall proceed as follows. First, one shall introduce a large class of systems which includes system (9) and where the expression of the output only depends on the system state and hence does not explicitly depend on the noise. A high gain observer that copes with the peaking phenomenon, referred to as NPHGO, will be designed for this class of systems and the main properties of the underlying observation error will be put forward. Then, one shall come back to system (9) to prove that it is included in the considered large class of systems and hence the proposed design of the NPHGO can be mimed leading thereby to a NPFHGO which copes not only with the peaking phenomenon problem but also with the observer sensitivity to noise.

Let us now consider the following class of systems which shall be used to design the NPHGO

$$
\left\{\begin{array}{l}
\dot{x}=A_{n} x+\varphi(u, x)+B_{n, n} \varepsilon(t)+B_{n, i_{w}} w(t) \\
y=C_{n} x=x_{1}
\end{array}\right.
$$

where $1 \leq i_{w} \leq n-1, B_{n, i_{w}} \in \mathbb{R}^{n}$ is a defined as in (3) with $k=n$ and $k_{0}=i_{w} ; w:[0,+\infty[\mapsto \mathbb{R}$ is a time varying unknown function the consideration of which shall be motivated later. All the other variables involved in system (10) keep the same meaning as in system (9). Notice that to avoid the redundance of variables, the same notation is used for the state variables and dimensions of systems (9) and system (10), i.e. $x$ and $n$. However, both systems are different since they assume different structures and the fact that the class of systems (10) includes systems (9) will be detailed later. Let us now focus on system (10).

The NPHGO observer design will be performed under the following usual assumptions.

A1. The state $x(t)$ and the control $u(t)$ are bounded, i.e. $x(t) \in X$ and $u(t) \in U$ where $X \subset \mathbb{R}^{n}$ and $U \subset$ $\mathbb{R}$ are compact sets. More precisely, there exists a positive constant $\rho_{M}$, such that $\forall t \geq 0,\left|x_{i}(t)\right| \leq$ $\rho_{M}, i=1, \ldots, n$.

A2. The functions $\varphi_{k}(u, \underline{x})$ for $k=1, \ldots, n$ are Lipschitz on $X$ with respect to $x$ uniformly in $u$, i.e. for $k=1, \ldots, n$, one has

$$
\begin{aligned}
& \exists L_{k}>0 ; \forall u \in U ; \forall(\hat{x}, x) \in X \times X, \\
& \left|\varphi_{k}\left(u, \underline{\hat{x}}_{k}\right)-\varphi_{k}\left(u, \underline{x}_{k}\right)\right| \leq L_{k} \sum_{i=1}^{k}\left|\tilde{x}_{i}\right|,
\end{aligned}
$$

where $\tilde{x}_{i}=\hat{x}_{i}-x_{i}$.
A3. The unknown function $\varepsilon$ is essentially bounded, i.e.

$$
\exists \delta_{\varepsilon}>0 ; \operatorname{Ess} \sup _{t \geq 0}|\varepsilon(t)| \leq \delta_{\varepsilon} .
$$

A4. The unknown function $w$ is essentially bounded, i.e.

$$
\exists \delta_{w}>0 ; \quad \text { Ess } \sup _{t \geq 0}|w(t)| \leq \delta_{w} .
$$

Since one will use saturation technics to cope with the peaking phenomenon, let us define the following saturation function

$$
\begin{aligned}
\forall z \in \mathbb{R}, \quad \operatorname{sat}_{r}(z) & =r \operatorname{sat}(z / r) \\
& =\left\{\begin{array}{ll}
z & \text { if }|z| \leq r \\
r \operatorname{sign}(z) & \text { if }|z|>r
\end{array},\right.
\end{aligned}
$$

where $r>0$ is a positive real and $\operatorname{sign}(\cdot)$ is the usual signum function.

Let

$R>\rho_{M}$,

be a positive real where $\rho_{M}$ is defined as in Assumption A1, be a positive real. One saturates the system nonlinearities $\varphi_{k}$ 's for $k=1, \ldots, n$ as follows

$\varphi_{i}^{s}\left(u, x_{1}, \ldots, x_{i}\right)=\varphi_{i}\left(u, \operatorname{sat}_{R}\left(x_{1}\right), \ldots, \operatorname{sat}_{R}\left(x_{i}\right)\right)$.

According to Assumption A2 and from the fact that the saturation function (14) is globally Lipschitz with a Lipschitz constant equal to 1 , the functions $\varphi_{i}^{s}$, s for $i=1, \ldots, n$ satisfy

$$
\begin{aligned}
& \forall u \in U ; \quad \forall(\hat{x}, x) \in \mathbb{R}^{n} \times \mathbb{R}^{n}, \quad \mid \varphi_{k}^{s}\left(u, \hat{x}_{1}, \ldots, \hat{x}_{k}\right) \\
& \quad-\varphi_{k}^{s}\left(u, x_{1}, \ldots, x_{k}\right)\left|\leq L_{k} \sum_{i=1}^{k}\right| \tilde{x}_{i} \mid .
\end{aligned}
$$

A constant bound can be derived for $\mid \varphi_{k}^{s}\left(u, \hat{x}_{1}, \ldots, \hat{x}_{k}\right)$ $-\varphi_{k}^{s}\left(u, x_{1}, \ldots, x_{k}\right) \mid$. Indeed, one has

$$
\begin{aligned}
& \left|\varphi_{k}^{s}\left(u, \hat{x}_{1}, \ldots, \hat{x}_{k}\right)-\varphi_{k}^{s}\left(u, x_{1}, \ldots, x_{k}\right)\right| \\
& \quad \leq L_{k} \sum_{i=1}^{k}\left|\operatorname{sat}_{R}\left(\hat{x}_{i}\right)-\operatorname{sat}_{R}\left(x_{i}\right)\right| \leq 2 L_{k} \sum_{i=1}^{k} R \\
& \quad \leq 2 n L_{\varphi} R .
\end{aligned}
$$

where

$$
L_{\varphi}=\max _{1 \leq k \leq n} L_{k}
$$

Two upper bounds can be derived for the norm of the error vector of the saturated nonlinearities 
$\tilde{\varphi}^{s}(u, \hat{x}, x) \triangleq \varphi^{s}(u, \hat{x})-\varphi^{s}(u, x)$ using either inequality (17) or (18). Indeed, the first bound can be derived using (17) as follows

$$
\begin{aligned}
\left|\tilde{\varphi}_{k}^{s}(u, \hat{x}, x)\right| & \leq L_{\varphi} \sum_{i=1}^{k}\left|\tilde{x}_{i}\right| \leq L_{\varphi} \sum_{i=1}^{n}\left|\tilde{x}_{i}\right| \\
& \leq \sqrt{n} L_{\varphi}\|\tilde{x}\|, \quad k=1, \ldots, n,
\end{aligned}
$$

and hence

$$
\left\|\tilde{\varphi}^{s}(u, \hat{x}, x)\right\| \leq n L_{\varphi}\|\tilde{x}\| .
$$

A constant bound can be derived using (18). Indeed, one has

$$
\begin{aligned}
\left\|\tilde{\varphi}^{s}(u, \hat{x}, x)\right\| & \leq \sqrt{n} \max _{1 \leq k \leq n}\left|\tilde{\varphi}_{k}^{s}(u, \hat{x}, x)\right| \\
& \leq 2 n \sqrt{n} L_{\varphi} R .
\end{aligned}
$$

Now, one postulates that the observer redesign can be handled for the following class of systems.

$$
\left\{\begin{array}{l}
\dot{x}=A_{n} x+\varphi^{s}(u, x)+B_{n, n} \varepsilon(t)+B_{n, i_{w}} w(t) \\
y=C_{n} x=x_{1}
\end{array}\right.
$$

where $\varphi^{s}(u, x)=\left(\varphi_{1}^{s}\left(u, x_{1}\right) \ldots \varphi_{n}^{s}(u, x)\right)^{T}$. Indeed, since system (10) coincides with system (23) on $X$, the observer that shall be designed for system (23) could be used in order to estimate the trajectories of system (10) which always lie in $X$. A class of MIMO systems which coincides with system (23) in the single output case and in the absence of $w(t)$ has been considered in [23] where a SHGO has been designed for this system. The observer equations for system (23) specialize as follows

$\dot{\hat{x}}=A_{n} \hat{x}+\varphi^{s}(u, \hat{x})-\theta \Delta_{n}^{-1}(\theta) \Gamma_{n}\left(C_{n} \hat{x}-y\right), \hat{x}\left(t_{0}\right) \in X$,

where $\hat{x} \in \mathbb{R}^{n}$ denotes the state estimate, $\Gamma_{n}$ is defined as in (4) with $k=n$ and the underlying coefficients $\gamma_{i}$ 's, $i=1, \ldots, n$ are chosen such that the matrix $\bar{A}_{n}=$ $A_{n}-\Gamma_{n} C_{n}$ is Hurwitz; $\Delta_{n}(\theta)$ is a diagonal matrix defined as in (6) with $k=n$ and $\theta \geq 1$.

Let $\tilde{x}=\hat{x}-x$ be the observation error associated to the SHGO (24). From (23) and (24), one has

$$
\begin{aligned}
\dot{\tilde{x}}= & A_{n} \tilde{x}+\tilde{\varphi}^{s}(u, \hat{x}, x)-\theta \Delta_{n}^{-1}(\theta) \Gamma_{n} C_{n} \tilde{x} \\
& -B_{n, n} \varepsilon(t)-B_{n, i_{w}} w(t),
\end{aligned}
$$

where $\tilde{\varphi}^{s}(u, \hat{x}, x)=\varphi^{s}(u, \hat{x})-\varphi^{s}(u, x)$. Proceeding as in [23], one can straightforwordly show that the observation error vectors $\underline{\tilde{x}}_{k}(t)$ for $k=1, \ldots, n$, where $\underline{\tilde{x}}_{k}$ is defined as in (7), satisfy the following property $\exists \theta_{0}>0 ; \quad \forall \theta \geq \theta_{0} ; \forall u \in U ; \forall \hat{x}\left(t_{0}\right) \in X ; \forall t \geq t_{0}$,$$
\left\|\underline{\tilde{x}}_{k}(t)\right\| \leq \sigma_{P_{n}}\left(\theta^{k-1} e^{-\beta_{n} \theta\left(t-t_{0}\right)}\left\|\tilde{x}\left(t_{0}\right)\right\|\right.
$$$$
\left.+\frac{\delta_{\varepsilon}}{\beta_{n} \theta^{n+1-k}}+\frac{\delta_{w}}{\beta_{n} \theta^{i_{w}+1-k}}\right),
$$

with

$\beta_{n}=\frac{\mu_{n}}{2 \lambda_{P_{n}}^{(M)}}$,

and where the SPD matrix $P_{n}$ and the positive real $\mu_{n}$ are given by equation (8) with $k=n$ and $\mathcal{A}_{n}=\bar{A}_{n}$ given by (4), $\delta_{\varepsilon}$ and $\delta_{w}$ are the essential upper bounds of $|\varepsilon(t)|$ and $|w(t)|$ given in Assumptions A3 and A4 respectively, $\theta_{0}=\max \left(1, n L_{\varphi} / \beta_{n}\right), L_{\varphi}$ is the Lipschitz constant given by (18) and $\sigma_{P_{n}}$ is the conditioning number of the matrix $P_{n}$.

Remark 2.1 According to inequality (26), in the absence of the unknown function $w(t)$, i.e. when $\delta_{w}=0$, the asymptotic observation errors related to $\underline{\tilde{x}}_{k}, k=$ $1, \ldots, n$, can be made as small as desired by choosing $\theta$ sufficiently high. Indeed, in this case, the underlying asymptotic ultimate bounds is equal to $\frac{\delta_{\varepsilon}}{\beta_{n} \theta^{n+1-k}}$. Since $k \leq n$, this bound is always lower or equal than $\frac{\delta_{\varepsilon}}{\beta_{n} \theta}$ which indeed tends to 0 when $\theta$ goes to $\infty$. In the case where $\delta_{w} \neq 0$, the above property is no longer true unless an additional constraint is made on $\delta_{w}$. Indeed, if $\delta_{w} \neq 0$, then one can easily check that the resulting asymptotic ultimate bound can be made as small as desired if the following condition on $\delta_{w}$ is satisfied

$\exists \bar{\delta}_{w}>0 ; \quad \delta_{w} \leq \frac{\bar{\delta}_{w}}{\theta^{n-i_{w}}}$,

where $\theta>\theta_{0}$. More precisely, under condition (28), inequality (26) specializes as follows

$$
\left\|\underline{\tilde{x}}_{k}(t)\right\| \leq \sigma_{P_{n}}\left(\theta^{k-1} e^{-\beta_{n} \theta\left(t-t_{0}\right)}\left\|\tilde{x}\left(t_{0}\right)\right\|+\frac{\delta_{\varepsilon}+\bar{\delta}_{w}}{\beta_{n} \theta^{n+1-k}}\right),
$$

Recall that in condition (28) the variable $n$ denotes the dimension of the considered class of systems and the variable $i_{w}$ is the rank of the state component the dynamics of which explicitly depends on $w(t)$.

The above SHGO (24) particularly suffers from the peaking phenomenon which takes place along the transient periods. In the following, one will show how to address the underlying problem appropriately. 


\section{Design of NPHGO}

The NPHGO is a redesigned version of the SHGO (24), which significantly reduces the peaking of the observer state variables during the transient periods. Such a performance improvement is achieved by a suitable modification of the observer through the consideration of adequate saturation functions as it shall be detailed later, after providing the observer equations. Before giving the equations of the proposed observer, one introduces the following definition which is needed for the observer design.

Definition 3.1 Let $A_{n}$ and $\Gamma_{n}$ be respectively the $n \times n$ matrix and the $n$ column vector defined as in (1) and (4) with $k=n$. The $n$ coefficients $\gamma_{i}$ 's of the vector $\Gamma_{n}, i=1, \ldots, n$ are said to satisfy the strong stability requirement with respect to $A_{n}$ if and only if for each $k \in\{1, \ldots, n\}$, the matrix $\bar{A}_{k}$ defined as in (4) is Hurwitz.

Notice that a similar notion has been introduced in [20] but the structures of the matrices $\bar{A}_{k}$ are different from those given by (4). Moreover, it is worth mentioning that some algorithms allowing to specify the appropriate $\gamma_{i}$ 's, $i=1, \ldots, n$, according to the strong stability requirement given in Definition 3.1, are available in [24]. An approach based on one of these algorithms shall be proposed in Sect. 3.2.

\subsection{Equations of the observer}

The equations of the proposed NPHGO can be written as follows

$\dot{\hat{x}}=A_{n} \hat{x}+\varphi^{s}(u, \hat{x})-\theta H\left(\tilde{x}_{1}\right), \quad \hat{x}\left(t_{0}\right) \in X$,

where $\hat{x}=\left(\hat{x}_{1} \ldots \hat{x}_{n}\right)^{T} \in \mathbb{R}^{n}, \tilde{x}_{1}=\hat{x}_{1}-$ $x_{1}, \theta>0$ is a design parameter and $H\left(\tilde{x}_{1}\right)=$ $\left(H_{1}\left(\tilde{x}_{1}\right) \ldots H_{n}\left(\tilde{x}_{1}\right)\right)^{T} \in \mathbb{R}^{n}$ with $H_{i}\left(\tilde{x}_{1}\right) \in \mathbb{R}$ for $i=1, \ldots, n$, is defined as follows

$$
\begin{aligned}
H_{1}\left(\tilde{x}_{1}(t)\right)= & \operatorname{sat}_{v}\left(\gamma_{1} \tilde{x}_{1}(t)\right) \text { and } \\
H_{i}\left(\tilde{x}_{1}(t)\right)= & \operatorname{sat}_{v}\left(\theta \frac{\gamma_{i}}{\gamma_{i-1}} H_{i-1}\left(\tilde{x}_{1}(t)\right)\right) \text { for } \\
& i=2, \ldots, n .
\end{aligned}
$$

where the $\gamma_{i}$ 's, $i=1, \ldots, n$ satisfy the strong stability requirement with respect to $A_{n}$, given in Definition 3.1; $\theta$ is a design parameter and is chosen sufficiently high in particular to satisfy $\theta>\frac{\gamma_{i-1}}{\gamma_{i}}, i=2, \ldots, n ; v>0$ is also a design parameter the choice of which shall be discussed later.

According to the expressions of the corrective terms $H_{i}$ 's, $i=1, \ldots, n$ and bearing in mind the definition of the saturation function given by (14), one can check that the expressions of these terms depend on the magnitude of $\left|\tilde{x}_{1}(t)\right|$ and they can be expressed as follows

- $\left|\tilde{x}_{1}(t)\right|>\frac{v}{\gamma_{1}} \Longrightarrow H\left(\tilde{x}_{1}(t)\right)$

$$
=v \operatorname{sign}\left(\tilde{x}_{1}(t)\right) U_{n}
$$

- $\left|\tilde{x}_{1}(t)\right| \leq \frac{v}{\gamma_{n} \theta^{n-1}} \Longrightarrow H\left(\tilde{x}_{1}(t)\right)$

$$
=\Delta_{n}^{-1}(\theta) \Gamma_{n} \tilde{x}_{1}(t),
$$

- $\forall k \in\{1, \ldots, n-1\}$,

$$
\begin{aligned}
& \frac{v}{\gamma_{k+1} \theta^{k}}<\left|\tilde{x}_{1}(t)\right| \leq \frac{v}{\gamma_{k} \theta^{k-1}} \Longrightarrow \\
& H\left(\tilde{x}_{1}(t)\right)=\left(\begin{array}{c}
\Delta_{k}^{-1}(\theta) \Gamma_{k} \tilde{x}_{1}(t) \\
v \operatorname{sign}\left(\tilde{x}_{1}(t)\right) U_{n-k}
\end{array}\right),
\end{aligned}
$$

where $\left(\Gamma_{n}, \Gamma_{k}\right),\left(U_{n}, U_{n-k}\right)$ and $\left(\Delta_{k}, \Delta_{n}\right)$ are given by (4), (5) and (6), respectively.

Before stating the results which put forward the main properties of observer (30), one shall introduce some definitions and notations which shall be used throughout the analysis of the observer properties. Indeed, one defines the following partition $\left\{\mathcal{S}_{k}\right\}_{-1 \leq k \leq n-1}$ on the interval [0,2R] where $R$ is defined as in (15),

$$
\begin{aligned}
\mathcal{S}_{-1} & \left.=] \frac{v}{\gamma_{1}}, 2 R\right], \\
\mathcal{S}_{k} & \left.=] \frac{v}{\gamma_{k+2} \theta^{k+1}}, \frac{v}{\gamma_{k+1} \theta^{k}}\right], \\
k & =0, \ldots, n-2 \text { and } \\
\mathcal{S}_{n-1} & =\left[0, \frac{v}{\gamma_{n} \theta^{n-1}}\right] .
\end{aligned}
$$

Each $\mathcal{S}_{k}$ shall be referred to as a sector. For each $k \in\{-1, \ldots, n-2\}, \mathcal{S}_{k+1}$ is the lower sector of $\mathcal{S}_{k}$. Similarly, for each $k \in\{0, \ldots, n-1\}, \mathcal{S}_{k-1}$ is the upper sector of $\mathcal{S}_{k}$. In the remaining of this section, one assumes without loss of generality that $\left|\tilde{x}_{1}\left(t_{0}\right)\right| \in \mathcal{S}_{-1}$.

Now, one shall put forward the time instants at which $\left|\tilde{x}_{1}(t)\right|$ enters and leaves the different sectors $\mathcal{S}_{k}$ since the initial time instant $t_{0}$ by setting the following definitions (see Fig. 1).

- If $\left|\tilde{x}_{1}(t)\right|$ leaves $\mathcal{S}_{k-1}$ and enters $\mathcal{S}_{k}$ for $k=$ $0, \ldots, n-1$, for the $i$ 'th time, at a finite time instant, then such a time instant shall be denoted by $t_{k-1, k}^{(i)}$ 


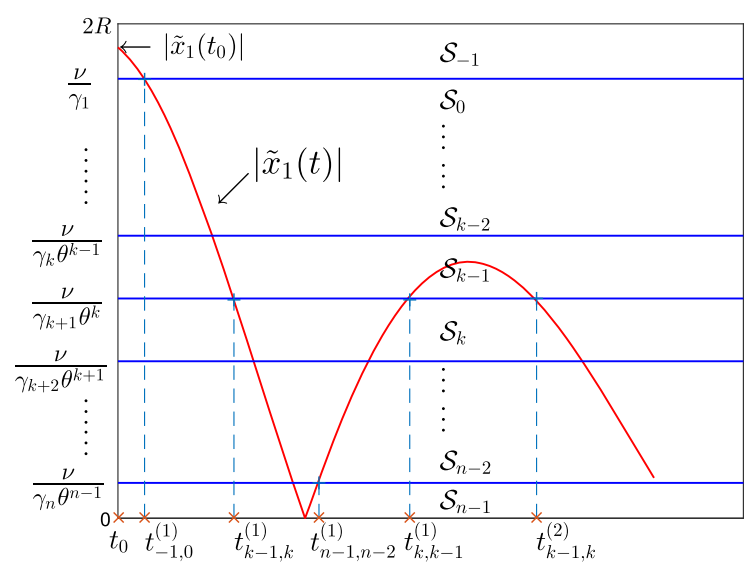

Fig. 1 Evolution of $\left|\tilde{x}_{1}(t)\right|$ through the sectors $\mathcal{S}_{k}$ with the associated time instants of crossing

and one shall state that $t_{k-1, k}^{(i)}$ exists. According to this definition, one has

$\left|\tilde{x}_{1}\left(t_{k-1, k}^{(i)-}\right)\right|>\frac{v}{\gamma_{k+1} \theta^{k}}$ and $\left|\tilde{x}_{1}\left(t_{k-1, k}^{(i)}\right)\right|=\frac{v}{\gamma_{k+1} \theta^{k}}$,

with $t_{k-1, k}^{(i)-}=\lim _{h \rightarrow 0}\left(t_{k-1, k}^{(i)}-h\right)$ where $h>0$ is a positive real. More precisely, the time instant $t_{k-1, k}^{(i)}$ is the time instant at which $\left|\tilde{x}_{1}(t)\right|$ enters $\mathcal{S}_{k}$.

- Similarly, if $\left|\tilde{x}_{1}(t)\right|$ leaves $\mathcal{S}_{k}$ and enters $\mathcal{S}_{k-1}$ for $k=0, \ldots, n-1$, for the $i$ 'th time at a finite time instant, then such a time instant shall be denoted by $t_{k, k-1}^{(i)}$ and one shall state that $t_{k, k-1}^{(i)}$ exists. Since $\left|\tilde{x}_{1}(t)\right|$ is continuous, one shall adopt the following definition,

$\left|\tilde{x}_{1}\left(t_{k, k-1}^{(i)}\right)\right|=\frac{v}{\gamma_{k+1} \theta^{k}}$ and $\left|\tilde{x}_{1}\left(t_{k, k-1}^{(i)+}\right)\right|>\frac{v}{\gamma_{k+1} \theta^{k}}$,

with $t_{k, k-1}^{(i)+}=\lim _{h \rightarrow 0}\left(t_{k, k-1}^{(i)}+h\right)$ where $h>0$ is a positive real. More precisely, the time instant $t_{k, k-1}^{(i)}$ is the time instant at which $\left|\tilde{x}_{1}(t)\right|$ leaves $\mathcal{S}_{k}$.

In the sequel, one shall state a set of propositions before giving the main theorem where the main property of the observation error associated to system (23) and observer (30) is given. In all these propositions, system (23) is assumed to satisfy Assumptions A1-A4.

Now, one states the following proposition the proof of which is given in Appendix A.

Proposition 3.1 Assume that $\left|\tilde{x}_{1}\left(t_{0}\right)\right| \in \mathcal{S}_{-1}$. Then,

(i) $t_{-1,0}^{(1)}>0$ exists with $\lim _{\theta \rightarrow \infty}\left(t_{-1,0}^{(1)}-t_{0}\right)=0$, (ii) $\exists \alpha_{-1,0, \theta}^{(1)}>0 ; \forall t \leq t_{-1,0}^{(1)},\|\tilde{x}(t)\| \leq \alpha_{-1,0, \theta}^{(1)}$ with $\lim _{\theta \rightarrow \infty}\left(\alpha_{-1,0, \theta}^{(1)} / \theta\right)=0$.

The behaviour of $\left|\tilde{x}_{1}(t)\right|$ for $t \geq t_{-1,0}^{(1)}$ is detailed by four propositions which are stated in what follows. The first of these propositions can be interpreted as a generalization of Proposition 3.1 since it details the evolving of $\left|\tilde{x}_{1}(t)\right|$ from the sector $\mathcal{S}_{-1}$ to the sector $\mathcal{S}_{n-1}$. Its statement is given below and its proof is given in Appendix B.

Proposition 3.2 Assume that $\left|\tilde{x}_{1}\left(t_{0}\right)\right| \in \mathcal{S}_{-1}$. Then, for any $k \in\{0, \ldots, n-1\}$, one has

(i) $t_{k-1, k}^{(1)}>0$ exists with $\lim _{\theta \rightarrow \infty}\left(t_{k-1, k}^{(1)}-t_{0}\right)=0$,

(ii) $\exists \alpha_{k-1, k, \theta}^{(1)}>0 ; \forall t \leq t_{k-1, k}^{(1)},\|\tilde{x}(t)\| \leq \alpha_{k-1, k, \theta}^{(1)}$ with $\lim _{\theta \rightarrow \infty}\left(\alpha_{k-1, k, \theta}^{(1)} / \theta\right)=0$.

According to Proposition 3.2, $\left|\tilde{x}_{1}(t)\right|$ will successively leave the sector $\mathcal{S}_{k-1}$ to enter $\mathcal{S}_{k}$ for $k=0, \ldots, n-1$. This in particular means that $\left|\tilde{x}_{1}(t)\right|$ will enter the sector $\mathcal{S}_{n-1}$ at the time instant $t_{n-2, n-1}^{(1)}$ and one has

$$
\begin{gathered}
\forall t \leq t_{n-2, n-1}^{(1)}, \quad\|\tilde{x}(t)\| \leq \alpha_{n-2, n-1, \theta}^{(1)} \quad \text { with } \\
\lim _{\theta \rightarrow \infty}\left(\alpha_{n-2, n-1, \theta}^{(1)} / \theta\right)=0 .
\end{gathered}
$$

The time evolution of $\left|\tilde{x}_{1}(t)\right|$ in the sector $\mathcal{S}_{n-1}$ is described in the following Proposition the proof of which is given in Appendix C.

Proposition 3.3 If $t_{n-1, n-2}^{(1)}$ exists and if the bound $\delta_{w}$ of $w(t)$ given by Assumption A4 satisfies condition (28), then one has

(i) $\lim _{\theta \rightarrow \infty} \tau_{n-1}^{(1)}=0$ where $\tau_{n-1}^{(1)}=t_{n-1, n-2}^{(1)}-$ $t_{n-2, n-1}^{(1)}$,

(ii) $\exists \alpha_{n-1, n-2, \theta}^{(1)} ; \forall t \leq t_{n-1, n-2}^{(1)},\|\tilde{x}(t)\| \leq \alpha_{n-1, n-2, \theta}^{(1)}$ with $\lim _{\theta \rightarrow \infty}\left(\alpha_{n-1, n-2, \theta}^{(1)} / \theta\right)=0$.

Notice that the statement in Proposition 3.3 deals with the case where $t_{n-1, n-2}^{(1)}$ exists. The non existence of such a time instant means that for $t \geq t_{n-2, n-1}^{(1)}$, $\left|\tilde{x}_{1}(t)\right|$ still evolve in $\mathcal{S}_{n-1}$ and never leaves it. In such a case and according to (33), one will have for all $t \geq t_{n-2, n-1}^{(1)}, H\left(\tilde{x}_{1}\right)=\Delta_{n}^{-1} \Gamma_{n} \tilde{x}_{1}$ and the equations of observer (30) will coincide with those of the SHGO (24). If the time instant $t_{n-1, n-2}^{(1)}$ exists, i.e. $\left|\tilde{x}_{1}(t)\right|$ leaves $\mathcal{S}_{n-1}$ and enters $\mathcal{S}_{n-2}$, then according to Proposition 3.3, the time period of the staying of $\left|\tilde{x}_{1}(t)\right|$ in $\mathcal{S}_{n-1}$ tends to zero when $\theta$ goes 
to infinity. Moreover, the vector of observation error remains bounded by $\alpha_{n-1, n-2, \theta}^{(1)}$ for all $t \leq t_{n-1, n-2}^{(1)}$ with $\lim _{\theta \rightarrow \infty}\left(\alpha_{n-1, n-2, \theta}^{(1)} / \theta\right)=0$. The behaviour of $\left|\tilde{x}_{1}(t)\right|$ for $t>t_{n-1, n-2}^{(1)}$ is detailed in the following proposition the proof of which is given in Appendix D.

Proposition 3.4 If $t_{n-1, n-2}^{(1)}$ exists and if the bound $\delta_{w}$ of $w(t)$ given by Assumption A4 satisfies condition (28), then there exists $k \in\{1, \ldots, n-1\}$ such that

(i) $t_{k-1, k}^{(2)}$ exists with $\lim _{\theta \rightarrow \infty} \tau_{k-1}^{(1)}=0$ where $\tau_{k-1}^{(1)}=$ $t_{k-1, k}^{(2)}-t_{k, k-1}^{(1)}$,

(ii) $\exists \alpha_{k-1, \theta}^{(1)}>0 ; \forall t \leq t_{k-1, k}^{(2)},\|\tilde{x}(t)\| \leq \alpha_{k-1, \theta}^{(1)}$ with $\lim _{\theta \rightarrow \infty}\left(\alpha_{k-1, \theta}^{(1)} / \theta\right)=0$.

According to Proposition 3.4 and in the case where $t_{n-1, n-2}^{(1)}$ exists, there exists a sector $\mathcal{S}_{k-1}$ with $k \in$ $\{1, \ldots, n-1\}$ which is such that, when $\left|\tilde{x}_{1}(t)\right|$ reaches and enters this sector (at the time instant $t_{k, k-1}^{(1)}$ ), it still evolve in this sector during a time period which tends to 0 when $\theta$ goes to infinity. After this time period, $\left|\tilde{x}_{1}(t)\right|$ does not enter the upper sector $\mathcal{S}_{k-2}$ but it comes back to the lower sector $\mathcal{S}_{k}$ at the time instant $t_{k-1, k}^{(2)}$. Recall that according to the adopted notations, $t_{k-1, k}^{(2)}$ corresponds to the time instant at which $\left|\tilde{x}_{1}(t)\right|$ leaves $\mathcal{S}_{k-1}$ and enters $\mathcal{S}_{k}$ for the second time bearing in mind that the existence of $t_{k-1, k}^{(1)}$ (i.e. the time instant where the same scenario occurred for the first time) has already been established by Proposition 3.2. An illustration of Proposition 3.4 is given in Fig. 1.

Remark 3.1 According to Proposition $3.4, t_{k-1, k}^{(2)}$ may exist for $k \in\{1, \ldots, n-1\}$. It is indeed shown in the proof of this proposition that $k$ cannot be equal to zero, i.e. $t_{-1,0}^{(2)}$ does not exist. Otherwise said, it is shown that $\left|\tilde{x}_{1}(t)\right|$ never come back to $\mathcal{S}_{-1}$ after the time instant $t_{-1,0}^{(1)}$. This also means the sequence $\left\{t_{-1,0}^{(i)}\right\}_{i \geq 1}$ is finite and reduced to $t_{-1,0}^{(1)}$.

Remark 3.2 It should be emphasized that the situation of $\left|\tilde{x}_{1}(t)\right|$ at the time instant $t_{k-1, k}^{(2)}$ is similar to its situation at the time instant $t_{k-1, k}^{(1)}$. Hence, the time evolution of $\left|\tilde{x}_{1}(t)\right|$ since the time instant $t_{k-1, k}^{(2)}$ is similar to its evolution after the time instant $t_{k-1, k}^{(1)}$ which has been detailed in Propositions 3.2-3.4 . According to these Propositions and bearing in mind Remark 3.1, $\left|\tilde{x}_{1}(t)\right|$ will evolve in the sectors $\mathcal{S}_{0}, \ldots, \mathcal{S}_{n-1}$ while $\|\tilde{x}(t)\|$ remains bounded. More precisely, one has a. For any $t \geq t_{-1,0}^{(1)},\left|\tilde{x}_{1}(t)\right|$ evolves in one of the sectors $\mathcal{S}_{0}, \ldots, \mathcal{S}_{n-1}$. Moreover, the time period of the staying of $\left|\tilde{x}_{1}(t)\right|$ in each of these sectors tends to 0 when $\theta$ goes to infinity.

b. For any finite time $T>0$,

$$
\begin{aligned}
\exists \alpha_{\theta}>0 ; \quad \forall t & \leq T, \quad\|\tilde{x}(t)\| \leq \alpha_{\theta} \quad \text { with } \\
\lim _{\theta \rightarrow \infty}\left(\alpha_{\theta} / \theta\right) & =0 .
\end{aligned}
$$

In the sequel, one shall show that there exists a time instant at which $\left|\tilde{x}_{1}(t)\right|$ enters for the last time the sector $\mathcal{S}_{n-1}$ and never leaves it. Notice that, since this time instant, the equations of observer (30) coincide with those of the SHGO (24).

One now states the main Proposition the proof of which is given in Appendix E.

Proposition 3.5 If the bound $\delta_{w}$ of $w(t)$ given by Assumption A4 satisfies condition (28), then the sequence $\left\{t_{k-1, k}^{(i)}\right\}_{i \geq 1}$ is finite for any $k \in\{0, \ldots, n-1\}$.

Let us denote by $i_{k}$ the number of terms in the sequence $\left\{t_{k-1, k}^{(i)}\right\}_{i \geq 1}$. According to Proposition 3.5, $i_{k}$ is finite for any $\vec{k} \in\{0, \ldots, n-1\}$. In particular, there exists a finite time instant $t_{n-2, n-1}^{\left(i_{n-1}\right)}$, such that for all $t \geq t_{n-2, n-1}^{\left(i_{n-1}\right)},\left|\tilde{x}_{1}(t)\right|$ never leaves $\mathcal{S}_{n-1}$. This means that, for $t \geq t_{n-2, n-1}^{\left(i_{n-1}\right)}$, the equations of observer (30) coincide with those of the SHGO (24).

On other aspects, since the time period of staying of $\left|\tilde{x}_{1}(t)\right|$ in each sector $\mathcal{S}_{k}$ for $k=-1, \ldots, n-1$ tends to 0 when $\theta$ tends to infinity, one deduces that $\lim _{\theta \rightarrow \infty}\left(t_{n-2, n-1}^{\left(i_{n-1}\right)}-t_{0}\right)=0$.

Remark 3.3 The result provided by Proposition 3.5, i.e. $i_{k}$ is finite, in particular means that for each $k=$ $1, \ldots, n$, there exists a time instant from which the $k$ first equations of the observer (30) coincide with their counterpart in observer (24) since each saturation function involved in the corrective term of (30) become equal to the identity function . The notion of strong stability requirement allowed to treat each subsystem composed by the underlying first $k$ equations as a perturbed systems and an appropriate ultimate bound is derived. When $k=n$, the overall system is recovered and observer (30) coincides with (24). Notice that and as stated before, the concept of strong stability requirement has been used in $[18,20]$ even though the underlying involved developments and arguments are quite different from those detailed in this paper. 
The main property of observer (30) directly results from the above results and it can be formulated in the following Theorem.

Theorem 3.1 Consider system (23) subject to Assumption $\mathrm{A} 1-\mathrm{A} 4$ together with observer (30) and assume that the bound $\delta_{w}$ of $w(t)$ given by Assumption A4 satisfies condition (28). Then, each component $\tilde{x}_{i}=$ $\hat{x}_{i}(t)-x_{i}(t)$ for $i=1, \ldots, n$, of the observation error satisfies the following property,

$$
\begin{aligned}
& \exists \theta^{\star}>0 ; \quad \forall \theta \geq \theta^{\star} ; \quad \exists t_{n-2, n-1}^{\left(i_{n-1}\right)}>0 ; \\
& \exists \alpha_{\theta}>0 ; \quad \forall u \in U ; \quad \forall \hat{x}\left(t_{0}\right) \in X, \quad \text { one has, } \\
& \text { (i) } \forall t \quad \text { s.t. } \quad t_{0} \leq t \leq t_{n-2, n-1}^{\left(i_{n-1}\right)},\left\|\tilde{x}_{i}(t)\right\| \leq \alpha_{\theta}, \\
& \text { (ii) } \forall t \geq t_{n-2, n-1}^{\left(i_{n-1}\right)},\left\|\tilde{x}_{i}(t)\right\| \\
& \leq \min \left\{\alpha_{\theta}, \sigma_{P_{n}}\left(\theta^{i-1} e^{-\beta_{n} \theta\left(t-t_{n-2, n-1}^{\left(i_{n-1}\right)}\right)} \alpha_{\theta}\right.\right. \\
& \left.\left.\quad+\frac{\delta_{\varepsilon}+\bar{\delta}_{w}}{\theta^{n+1-i} \beta_{n}}\right)\right\},
\end{aligned}
$$

where $\beta_{n}$ is defined by (27), $P_{n}$ is given by (8) with $k=$ $n$ and $\mathcal{A}_{k}=\bar{A}_{n}$ where $\bar{A}_{n}$ is defined as in (4) and $\delta_{\varepsilon}, \bar{\delta}_{w}$ are as defined by (12) and (28), respectively. Moreover, one has $\lim _{\theta \rightarrow \infty}\left(t_{n-2, n-1}^{\left(i_{n-1}\right)}-t_{0}\right)=\lim _{\theta \rightarrow \infty}\left(\alpha_{\theta} / \theta\right)$ $=0$.

Remark 3.4 The analysis of the time evolution of $\left|\tilde{x}_{1}(t)\right|$ carried out through Propositions $3.1-3.5$ has assumed that the initial condition $\left|\tilde{x}_{1}\left(t_{0}\right)\right| \in \mathcal{S}_{-1}$. In the case where $\left|\tilde{x}_{1}\left(t_{0}\right)\right| \in \mathcal{S}_{k^{\star}}$ where $k^{\star} \in\{0, \ldots, n-2\}$, one can show that the time evolution of $\left|\tilde{x}_{1}(t)\right|$ still be similar to its evolution in the case where $\left|\tilde{x}_{1}\left(t_{0}\right)\right| \in \mathcal{S}_{-1}$ up to the following minor difference. Indeed, by proceeding as in the proof of Proposition 3.2, one can show that $\left|\tilde{x}_{1}(t)\right|$ will first cross the lower sectors until entering in $\mathcal{S}_{n-1}$ as in the case where $\left|\tilde{x}_{1}\left(t_{0}\right)\right| \in \mathcal{S}_{-1}$. Then, if $\left|\tilde{x}_{1}(t)\right|$ leaves $\mathcal{S}_{n-1}$ to go to the upper sectors, it will reach the most upper sector and then come back to the lower ones as described in Proposition 3.4. Notice that the most upper sector may be $\mathcal{S}_{-1}$ and this was not possible when $\left|\tilde{x}_{1}\left(t_{0}\right)\right| \in \mathcal{S}_{-1}$. Now, it suffices to consider any time instant at which $\left|\tilde{x}_{1}(t)\right|$ evolves in the most upper sector and one can show that the time evolution of $\left|\tilde{x}_{1}(t)\right|$ since this time instant is similar to its evolution when $\left|\tilde{x}_{1}\left(t_{0}\right)\right| \in \mathcal{S}_{-1}$.

\subsection{Tuning the observer design parameters}

The corrective term of observer (30)-(31) involves three design parameters, the vector $\Gamma_{n}$ and the positive reals $\theta$ and $\nu$. As stated above, the $n$ coefficients $\gamma_{i}$ 's, of the vector $\Gamma_{n}$ have to satisfy the strong stability requirement with respect to $A_{n}$. An appropriate choice of these parameters can be achieved using one of the following two approaches to which one shall refer as Approach 1 and Approach 2.

Approach 1: Assign the poles of $\bar{A}_{n}$ to predefined values and compute the underlying $\Gamma_{n}$. This can be easily achieved for example by calling one of matlab standard functions 'acker' or 'place'. After the obtention of the $\gamma_{i}$ 's, check the full stability requirement by computing the roots of the $n-1$ polynomials $P_{k}(\lambda)=\lambda^{k}+\sum_{i=0}^{k-1} \gamma_{i} \lambda^{i}, k=1, \ldots, n-1$. The full stability requirement is fulfilled if all the roots of each polynomial have negative real parts.

Approach 2: This approach is based on Theorem 3 in [24] which provides a recursive algorithm allowing to generate the coefficients $\gamma_{i}$ 's that satisfy the full stability requirement. This algorithm can be described as follows: Let $\delta \approx 4.07959$ be the unique real root of $x^{3}-5 x^{2}+4 x-1=0$ and let $\alpha>0$ be a positive real. The values of the $\gamma_{i}$ 's, $i=1, \ldots, n$ are computed as follows:

$$
\begin{aligned}
& \gamma_{1}=\alpha, \quad \gamma_{2}=\frac{\alpha^{2}}{\sqrt{\delta}} \text { and } \\
& \gamma_{k}=\frac{\gamma_{k-1}^{2}}{\sqrt{\delta} \gamma_{k-2}}, \quad k=3, \ldots, n .
\end{aligned}
$$

At a first glance, the second approach seems more attractive than the first one since it allows to provide the appropriate coefficients $\gamma_{i}$ 's in a direct manner. However, the first approach has not to be systematically discarded. Indeed, when the dimension of the system is relatively low, say lower than 10 , the strong stability requirement is often satisfied when the coefficients $\gamma_{i}$ 's are chosen such that the matrix $\bar{A}_{n}$ is Hurwitz with predefined eigenvalues. This issue has been checked numerically in simulation by considering many sets of predefined eigenvalues for $\bar{A}_{n}$. For example, if all the eigenvalues of $\bar{A}_{n}$ are assigned to (-1), then the strong stability requirement is satisfied for all $n$ lower or equal to 11 .

The choice of the design parameters $\theta$ and $\nu$ has to be achieved by a trial an error approach. It is well known 
that in the case of the SGHO, relatively high values of $\theta$ allow a fast convergence of the observation error to zero but amplify the peaking phenomenon. Since the behaviour with respect to the peaking phenomenon has been significantly improved, the use of relatively high values for the parameter $\theta$ in observer (30) is no longer discouraged. Let us now focus on the level of saturation $v$ and assume that the value of $\theta$ is fixed. It is clear that for very high values of $v$, the peaking phenomenon still be present and the behaviour of the NPGHO becomes identical to that of the SHGO. However, very low values of $v$ may lead to longer transient periods of the observer. This means that the period of staying of the observation error $\tilde{x}_{1}(t)$ in each sector $S_{k}$ before leaving it becomes longer. More precisely, if $v$ is chosen such that the product $\nu \theta$ is not high enough, then the transient period becomes longer and the saturation process may fail [see Eq. (63)] and Eqs. [(76), (78)].

\section{Design of a NPFHGO}

Let us now come back to system (9) and let us show how one can design for this system a NPFHGO, i.e. an observer similar to (30) which allows to cope with the non peaking phenomenon as well as with the sensitivity to noise of the observer. Before detailing this issue, it should be emphasized that in the case where system (9) is noise free, i.e. $v(t)=0$, then it is easy to see that this system coincide with (10) where $w(t)=0$ and hence an observer similar to (30) can be designed for system (9). Let us now focus on the case where $v(t) \neq 0$, i.e. the system output is corrupted by the additive noise $v(t)$. One shall show how system (9) can be augmented by a multiple integrator of the corrupted output leading to an augmented system the structure of which similar to that of system (10). As a result a SHGO similar to observer (24) but involving filtering capabilities can be designed. Such observer is referred to as FHGO. Exploiting the fact the structure of the FHGO is similar to that of the SHGO, the redesign approach which led to the NPHGO can be used to derive a NPFHGO from the FHGO. One now propose to detail the deriving of the FHGO and the NPFHGO.

\subsection{Design of the FHGO}

Many approaches dealing with the observer designs have been proposed to reduce the underlying observers sensitivity to noise measurements either by appropriately updating the observer design parameter $\theta$ $[16,17,25]$ or by substituting in the observer corrective term the output observation error by an appropriate filtered version provided by a linear filter cascaded with the original system $[14,26,27]$. The main drawback of the approach where a linear filter is considered lies in the fact that the power of the design parameter $\theta$ involved in the resulting observer gain increases with an amount equal to the filter order and this leads to the amplification of the peaking phenomenon. Since the peaking phenomenon has already been coped with thanks to the saturation functions, one shall adopt the approach using a filtered version of the output observation error to deal with the observer sensitivity to high frequency signals. In [26], the considered linear filter is simply an integrator of the original system output. A multiple integrator of the system output has been considered in [27]. Proceeding as in [26,27] where interesting filtering properties for the underlying integral observers are recorded, one proposes to augment system (23) by a cascade of integrators of the output as follows

$\left\{\begin{array}{l}\dot{z}=A_{m} z+B_{m, m} y \\ \dot{x}=A_{n} x+\varphi^{s}(u, x)+B_{n, n} \varepsilon(t) \\ \bar{y}(t)=z_{1}(t)\end{array}\right.$

where $z^{T}=\left(z_{1} \ldots z_{m}\right) \in \mathbb{R}^{m}, A_{m}$ and $B_{m, m}$ are defined as in (1) and (3) with $k=k_{0}=m$. Notice that in order to improve the performance of the observer with respect to the output noise measurements, the output of the augmented system (41) is $z_{1}(t)$ which is an integrator of order $m$ of the original system noisy output $y(t)$. One shall show later, after giving the equations of the resulting observer, that the first $m$ equations of the observer can be interpreted as a linear first order filter that provides a filtered version of the output observation error which is used in the observer corrective term. Now, since $y=C_{n} x+v(t)$, system (41) can be written under the following form

$$
\left\{\begin{array}{c}
\left(\begin{array}{c}
\dot{z} \\
\dot{x}
\end{array}\right)=\left(\begin{array}{cc}
A_{m} & B_{m, m} C_{n} \\
0_{m \times n} & A_{n}
\end{array}\right)\left(\begin{array}{l}
z \\
x
\end{array}\right)+\left(\begin{array}{c}
0 \\
\varphi^{s}(u, x)
\end{array}\right) \\
+B_{n+m, n+m} \varepsilon(t)+B_{n+m, m} v(t) \\
\bar{y}=C_{n+m}\left(\begin{array}{c}
z \\
x
\end{array}\right)=z_{1}
\end{array}\right.
$$


where $B_{n+m, n+m}$ is as in (3) with $k=k_{0}=n+m$ and $B_{n+m, m}$ as in (3) with $k=n+m$ and $k_{0}=m$.

Taking into account the structure of the matrices $A_{m}, A_{n}$ and $B_{m}$, one can check that

$$
\left(\begin{array}{cc}
A_{m} & B_{m, m} C_{n} \\
0_{n \times m} & A_{n}
\end{array}\right)=A_{n+m},
$$

where $A_{n+m}$ is as in (1) with $k=n+m$.

According to the above equality, the augmented system (42) can be written under the following form

$$
\left\{\begin{array}{c}
\left(\begin{array}{c}
\dot{z} \\
\dot{x}
\end{array}\right)=A_{n+m}\left(\begin{array}{l}
z \\
x
\end{array}\right)+\left(\begin{array}{c}
0 \\
\varphi^{S}(u, x)
\end{array}\right) \\
+B_{n+m, n+m} \varepsilon(t)+B_{n+m, m} v(t) \\
\bar{y}=C_{n+m}\left(\begin{array}{c}
z \\
x
\end{array}\right)=z_{1}
\end{array}\right.
$$

where $C_{n+m}$ is as in (2) with $k=n+m$. It is clear that the structure of the $n+m$ dimensional system (44) is similar to that of the $n$ dimensional system (10) with $w(t)=v(t)$ and $i_{w}=m$. As a result, a SHGO can be designed for this system. The equations of the underlying SHGO can be written as follows

$$
\begin{aligned}
\left(\begin{array}{c}
\dot{\hat{z}} \\
\dot{\hat{x}}
\end{array}\right)= & A_{n+m}\left(\begin{array}{l}
\hat{z} \\
\hat{x}
\end{array}\right)+\left(\begin{array}{c}
0 \\
\varphi^{s}(u, \hat{x})
\end{array}\right) \\
& -\theta \Delta_{n+m}^{-1}(\theta) \Gamma_{n+m}\left(\hat{z}_{1}-z_{1}\right),
\end{aligned}
$$

where $\hat{z} \in \mathbb{R}^{m}$ and $\hat{x} \in \mathbb{R}^{n}$ are the respective estimates of $z$ and $x, \Delta_{n+m}$ is a diagonal matrix defined as in (6) and $\Gamma_{n+m}^{T}=\left(\gamma_{1} \ldots \gamma_{n+m}\right) \in \mathbb{R}^{n+m}$ where the coefficients $\gamma_{i}$ 's for $i=1, \ldots, n+m$ are positive reals satisfying the strong stability requirement with respect to $A_{n+m}$. Notice that, the equations of the above observer depend on the variable $z$ (or more precisely $z_{1}$ ) the dynamics of which is governed by the first $m$ scalar Ordinary Differential Equations (ODE) of system (41). Hence the number of scalar ODE's constituting the observer is equal to $(n+2 m)$. In order to reduce the number of these equations to $(n+m)$, one shall proceed as follows.

First, one can check the following equalities

$$
\begin{aligned}
& \Delta_{n+m}(\theta)=\operatorname{diag}\left(\Delta_{m}(\theta), \quad \frac{1}{\theta^{m}} \Delta_{n}(\theta)\right), \\
& \Gamma_{n+m}=\left(\begin{array}{ll}
\Gamma_{m}^{T} & \Gamma_{m+1, n+m}^{T}
\end{array}\right)^{T},
\end{aligned}
$$

where $\Delta_{n+m}, \Delta_{m}$ and $\Delta_{n}$ are diagonal matrices defined as in (6), $\Gamma_{m}^{T}=\left(\begin{array}{lll}\gamma_{1} & \ldots & \gamma_{m}\end{array}\right)$ and $\Gamma_{m+1, n+m}^{T}=$ $\left(\gamma_{m+1} \ldots \gamma_{n+m}\right)$. Using equalities (43) and (46), the equations of observer (45) can be written under the following expanded form

$$
\left\{\begin{array}{l}
\dot{\hat{z}}=A_{m} \hat{z}+B_{m, m} \hat{x}_{1}-\theta \Delta_{m}^{-1}(\theta) \Gamma_{m}\left(\hat{z}_{1}-z_{1}\right) \\
\hat{\hat{x}}=A_{n} \hat{x}+\varphi^{s}(u, \hat{x})-\theta^{m} \Delta_{n}^{-1}(\theta) \Gamma_{m+1, n+m}\left(\hat{z}_{1}-z_{1}\right)
\end{array}\right.
$$

System (47) is constituted by two cascade subsystems where $\hat{z}$ and $\hat{x}$ are the respective states of these subsystems. It is clear that the time derivative of $\hat{x}$ is linked to that of $\hat{z}$ through the term $\hat{z}_{1}-z_{1}$, only. Hence, set $\eta=\hat{z}-z \in \mathbb{R}^{m}$ and let $\eta_{i}$ denotes the $i$ 'th component of $\eta$ for $i=1, \ldots, m$. Using the respective first equations of systems (41) and (47) as well as equalities (46), the equations of observer (47) can be written using the states $\eta$ and $\hat{x}$ as follows

$$
\begin{aligned}
\left(\begin{array}{c}
\dot{\eta} \\
\dot{\hat{x}}
\end{array}\right)= & \left(\begin{array}{c}
A_{m} \eta(t)+B_{m, m} C_{n} \hat{x}-B_{m} y \\
A_{n} \hat{x}+\varphi^{s}(u, \hat{x})
\end{array}\right) \\
& -\theta \Delta_{n+m}^{-1}(\theta) \Gamma_{n+m} \eta_{1} \\
= & A_{n+m}\left(\begin{array}{c}
\eta \\
\hat{x}
\end{array}\right)+\left(\begin{array}{c}
-B_{m, m} y \\
\varphi^{s}(u, \hat{x})
\end{array}\right) \\
& -\theta \Delta_{n+m}^{-1}(\theta) \Gamma_{n+m} \eta_{1}
\end{aligned}
$$

Notice that $\eta\left(t_{0}\right)=0$ is an appropriate initial value for the ODE governing $\eta$ since it reduces the transient period of the filter.

It is clear that system (48) which provides a smooth estimate, $\hat{x}$, of the state $x$ requires the resolution of $n+m$ scalar ODE's, only. The first $m$ ODE's are associated to a linear filter of order $m$ the entry of which is the noisy output observation error, i.e. $C_{n} \hat{x}(t)-y(t)$, and the resulting filtered output provided by this filter, i.e. $\eta_{1}=C_{m} \eta$, is then used in the correction term in both subsystems (overall observer). Moreover, since the structure of observer (48) is similar to that of the SHGO (24), then the same redesign process used to derive (30) from (24) can be used in a straightforward manner to derive the equations of a NPFGHO that inherits the main properties of the NPHGO together with those of the FHGO. Before giving the equations of the observer, let us derive an upper bound of the underlying observation error under the assumption that the output measurements noise $v(t)$ is essentially bounded by an essential bound $\delta_{v}$ satisfying a condition similar to (28). Bearing in mind the significance of the variables involved in (28) (see, Remark 2.1), the underlying condition can be obtained by substituting in (28), $n$ and $i_{w}$ by $(n+m)$ and $m$, respectively, i.e. condition (28) specializes as follows

$\exists \bar{\delta}_{v}>0 ; \delta_{v} \leq \frac{\bar{\delta}_{v}}{\theta^{(n+m)-m}}=\frac{\bar{\delta}_{v}}{\theta^{n}}$,

To summarize, the output measurements noise $v(t)$ is assumed to satisfy the following condition

$\exists \bar{\delta}_{v}>0 ; \quad$ Ess $\sup _{t \geq 0}|v(t)| \leq \frac{\bar{\delta}_{v}}{\theta^{n}}$ 
Let $\xi=\left(\begin{array}{l}\eta \\ \tilde{x}\end{array}\right)$ where $\tilde{x}=\hat{x}-x$ be the observation error associated to observer (48) and system (44). Then, each component of the observation error, $\xi_{i}, i=1, \ldots, m+n$, satisfies an inequality similar to (29). In particular, for $i=1, \ldots, n$, one has $\xi_{m+i}=\tilde{x}_{i}$ and it satisfies

$$
\begin{aligned}
& \exists \bar{\theta}_{0}>0 ; \quad \forall \theta \geq \bar{\theta}_{0} ; \quad \forall u \in U ; \quad \forall \hat{x}\left(t_{0}\right) \in X ; \quad \forall t \geq t_{0}, \\
& \left\|\underline{\tilde{x}}_{i}(t)\right\| \leq \sigma_{P_{n+m}}\left(\theta^{m+i-1} e^{-\beta_{n+m} \theta\left(t-t_{0}\right)}\left\|\tilde{x}\left(t_{0}\right)\right\|\right. \\
& \left.+\frac{\delta_{\varepsilon}+\bar{\delta}_{v}}{\theta^{n+1-i} \beta_{n+m}}\right),
\end{aligned}
$$

where $\beta_{n+m}$ is defined as in (27) by substituting $n$ by $n+m$, the SPD matrix $P_{n+m}$ is given by Eq. (8) with $k=n+m, \delta_{\varepsilon}$ and $\bar{\delta}_{v}$ are respectively given (12) and (50), $\bar{\theta}_{0}=\max \left(1,2(n+m) L_{\varphi} / \beta_{n+m}\right)$ and $L_{\varphi}$ is the Lipschitz constant as in inequality (26).

Notice that $\left\|\xi\left(t_{0}\right)\right\|$ has been substituted by $\left\|\tilde{x}\left(t_{0}\right)\right\|$ in the right side of inequality (51) since $\eta\left(t_{0}\right)=0$ and hence $\left\|\xi\left(t_{0}\right)\right\|=\left\|\tilde{x}\left(t_{0}\right)\right\|$. One also notices that the power of $\theta$ multiplying the decreasing exponential in (51) is $m+i-1$ and it was only $i-1$ in the bound associated with the SHGO given by the right side of inequality (29). This means that a drawback resulting from the adding of the filter is the amplification of the peaking phenomenon of the FHGO compared to the SHGO. Nevertheless, the increasing of this power has no significant impact since the underlying FHGO shall be redesigned into a NPFHGO.

Remark 4.1 Notice that condition (50) is very similar to the one derived in [20]. It is rather conservative since it requires that the essential bound of the output measurement noise has to be relatively small and of the order of $\theta^{-n}$ where $n$ is the dimension of the original system with noisy outputs. The non dependence of this condition with the order of the filter, $m$, is to emphasized.

\subsection{Equations of the NPFHGO}

As stated above, the redesign process that has been already used to derive the NPHGO (30) from its underlying SHGO (24), can be applied to the FHGO (48) to derive the NPFHGO the equations of which are then specialized as follows

$$
\left(\begin{array}{c}
\dot{\eta} \\
\hat{x}
\end{array}\right)=A_{n+m}\left(\begin{array}{l}
\eta \\
\hat{x}
\end{array}\right)+\Phi^{s}(u, \eta, \hat{x}, x)
$$

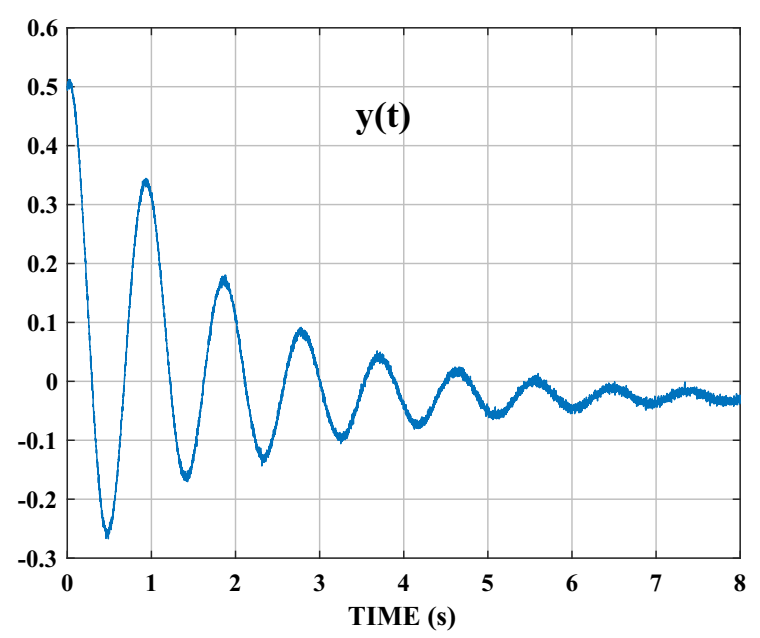

Fig. 2 Noisy measurements of $x_{1}$

$$
-\theta H\left(\eta_{1}\right), \quad \eta\left(t_{0}\right)=0, \hat{x}\left(t_{0}\right) \in X,
$$

where $\Phi^{s}(u, \eta, \hat{x}, x)=\left(\begin{array}{c}-B_{m, m} y \\ \tilde{\varphi}^{s}(u, \hat{x}, x)\end{array}\right), H\left(\eta_{1}\right) \in$ $\mathbb{R}^{n+m}$ and the corresponding components $H_{i}\left(\eta_{1}\right) \in$ $\mathbb{R}$, for $i=1, \ldots, n+m$, are defined as follows [compare with (31)]

$$
\begin{aligned}
& H_{1}\left(\eta_{1}\right)=\operatorname{sat}_{v}\left(\gamma_{1} \eta_{1}\right) \text { and } \\
& H_{i}\left(\eta_{1}\right)=\operatorname{sat}_{v}\left(\theta \frac{\gamma_{i}}{\gamma_{i-1}} H_{i-1}\left(\eta_{1}\right)\right) \text { for } i=2, \ldots, n .
\end{aligned}
$$

where the $\gamma_{i}$ 's, $i=1, \ldots, n+m$ satisfy the strong stability requirement with respect to $A_{n+m}$ given in Definition 3.1.

It should be emphasized that observer (52) simultaneously inherits the main properties of observer (30) in terms of a significant reducing of the peaking of the observer state variables as well as those of observer (48) in improving the performance of the observer sensitivity with respect to high frequency signals.

Remark 4.2 The extension of the NPFHGO design to the multioutput class of systems considered in [23] can be straightforwardly handled by simply applying the saturation process to each scalar output.

\section{Example}

In this section, the performance and main properties of the proposed NPFHGO are illustrated and compared 

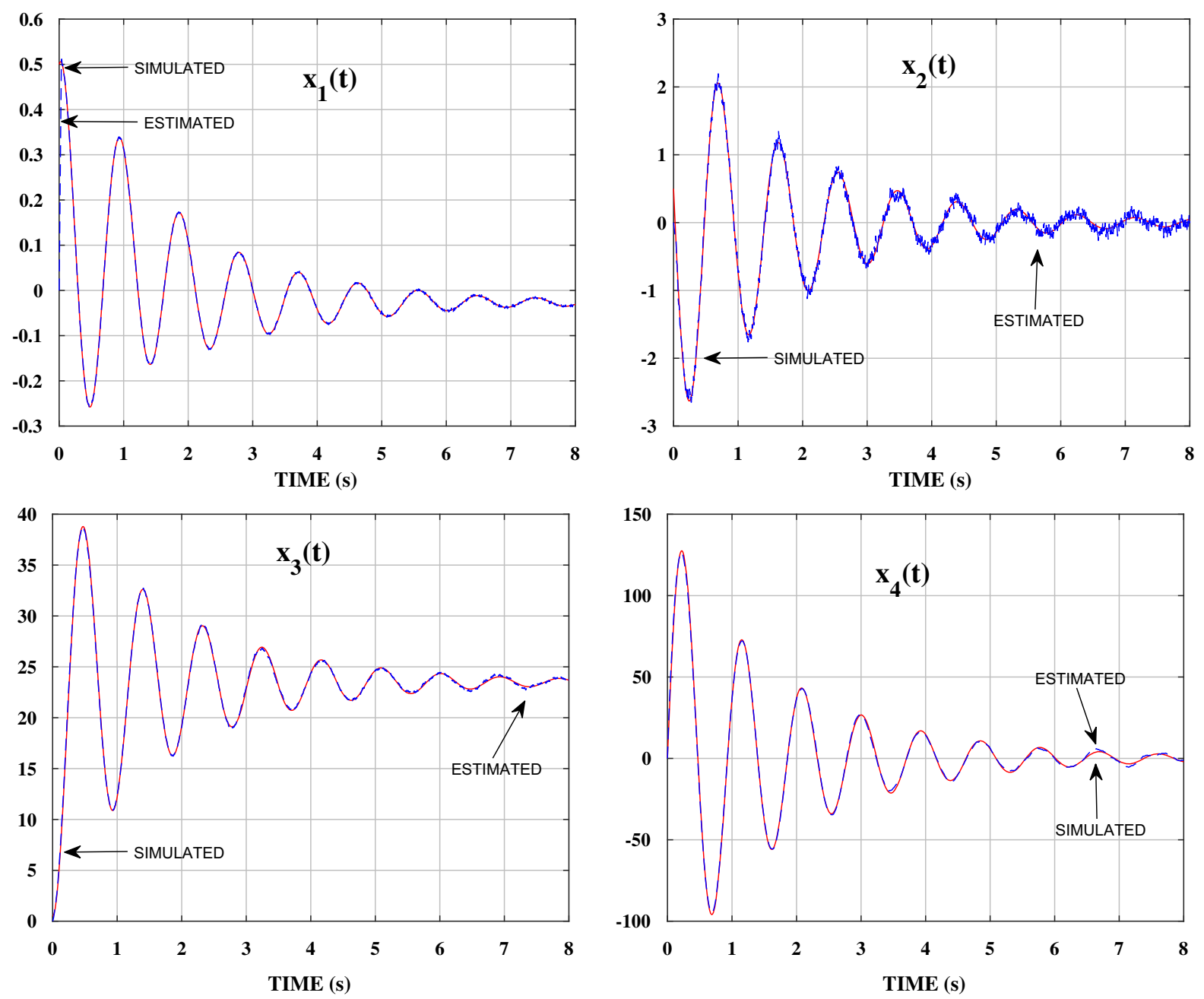

Fig. 3 State estimation with the NPFHGO

with that of the SHGO through an example involving a single-link robot arm introduced in [28] and used in [14]. The underlying mathematical model is described by the following differential equations

$$
\left\{\begin{aligned}
\dot{x}_{1}= & x_{2} \\
\dot{x}_{2}= & \bar{K} /\left(J_{2} N\right) x_{3}-\left(F_{2} / J_{2}\right) x_{2}-\left(\bar{K} / J_{2}\right) x_{1} \\
& -\left(\bar{m} g d / J_{2}\right) \cos \left(x_{1}\right) \\
\dot{x}_{3}= & x_{4} \\
\dot{x}_{4}= & \left(1 / J_{1}\right) u-\bar{K} /\left(J_{1} N\right) x_{1}-\bar{K} /\left(J_{2} N\right) x_{3} \\
& -\left(F_{1} / J_{1}\right) x_{4}+\varepsilon(t) \\
y= & x_{1}+v(t)
\end{aligned}\right.
$$

where $\varepsilon(t)$ and $v(t)$ denote respectively the disturbance and the measurement noise, $J_{1}, J_{2}, \bar{K}, N, \bar{m}, g, d$, $F_{1}$ and $F_{2}$ are the model parameters which are posi- tive constants. More specifically, the control sequence $\{u(t)\}$ is specified as in [28], i.e.

$$
\begin{aligned}
u= & \operatorname{sat}_{M}\left\{m g d J_{1} /\left(J_{2} N\right)-\left(J_{1} J_{2} N / \bar{K}\right)\left(L^{4} c_{1} x_{1}+L^{3} c_{2} x_{2}\right.\right. \\
& \left.\left.+L^{2} c_{3}\left(\bar{K} /\left(J_{2} N\right) x_{3}-\left(m g d / J_{2}\right)\right)+L c_{4} \bar{K} /\left(J_{2} N\right) x_{1}\right)\right\},
\end{aligned}
$$

with $M>0$ and $s a t_{M}$ defined as in (14), the $c_{i}$ 's, $i=1, \ldots, 4$ and $L$ are positive constants. Using the fact that $\bar{K} /\left(J_{2} N\right)=1$, it is easy to check that system (54) is under form (10) and as a result a NPFHGO (and a SHGO) observer(s) can be designed. Indeed, a NPFHGO of the form (52) has been designed with a filter of order 2, i.e. $m=2$. The parameters of system (54) are chosen as follows $F_{1}=0.1, F_{2}=0.15$, $J_{1}=0.15, J_{2}=0.2, \bar{K}=0.4, N=2, \bar{m}=0.8$, $g=9.81$ and $d=0.6$. The control $u(t)$ is com- 

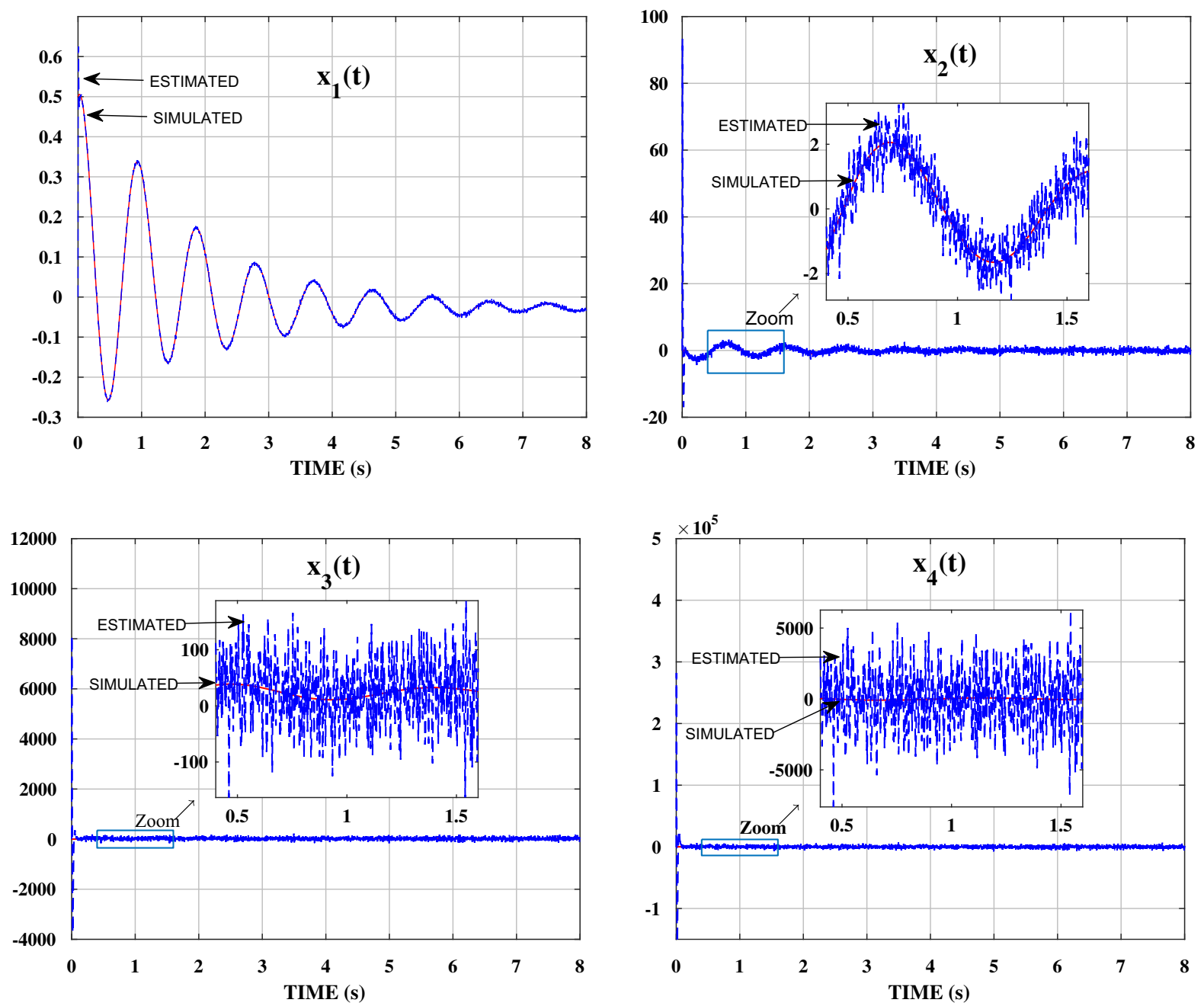

Fig. 4 State estimation with the SHGO

puted with the following parameters setting $c_{1}=4$, $c_{2}=7.91, c_{3}=6.026, c_{4}=1.716, L=3$ and $M=200$. In the simulation, the uncertainty has been chosen as $\varepsilon(t)=\sin (5 t)$, and the output $y(t)$ has been corrupted with a gaussian noise $v(t)$ with a zero mean value and a standard deviation equal to 0.004 as shown in Fig. 2. The initial conditions of system (54) and the observer have been chosen as in $[14,28]$, i.e. $x^{T}(0)=\left(\begin{array}{llll}0.5 & 0.5 & 0.5 & 0.5\end{array}\right)$ and $\hat{x}^{T}\left(\begin{array}{l}0\end{array}\right)=\left(\begin{array}{llll}0 & 0 & 0 & 0\end{array}\right)$. With the considered values for the initial conditions, the state variables of system (54) are bounded with $x_{1}(t) \in\left[\begin{array}{ll}-0.26 & 0.51\end{array}\right], x_{2}(t) \in\left[-2.632 .06, x_{3}(t) \in\right.$ $\left[\begin{array}{ll}0.5 & 38.8\end{array}\right]$ and $x_{4}(t) \in[-95.94127 .56]$. Hence, the value of the level $R$ to saturate the nonlinearities in the observer [see (16)] has to be chosen at least greater than the maximum absolute value of the above interval bounds, i.e. $R>\rho_{M}=127.56$. A general rule that can be adopted for choosing $R$ is to set it to an arbitrarily great value. Indeed, simulation results which are presented later have been obtained by setting $R=1000$. Other simulation with other values of $R\left(>\rho_{M}\right)$ have been carried out and they led to the same results.

The value of $\theta$ was set to $150, v$ to 0.1 and the coefficients $\gamma_{i}$ for $k=1, \ldots, 6$ are chosen such that the poles of $\bar{A}_{6}$ are located at -1 . It has been checked that the underlying value do satisfy the strong stability requirement. For comparison purposes, a SHGO under the form (24) has also been designed for system (54) and it has been simulated with a gain $\Gamma=\left(\begin{array}{llll}4 & 6 & 4 & 1\end{array}\right)^{T}$ in such a way that all the eigenvalues of the (linear part 
Table 1 Comparison of the peaking phenomenon between NPFHGO and SHGO

\begin{tabular}{lll}
\hline & NPFGHO & SHGO \\
\hline$\left\|\tilde{x}_{1}\right\|_{\infty}$ & 0.5 & 0.5 \\
$\left\|\tilde{x}_{2}\right\|_{\infty}$ & 0.5 & 92.9466 \\
$\left\|\tilde{x}_{3}\right\|_{\infty}$ & 0.6296 & $8.1410^{3}$ \\
$\left\|\tilde{x}_{4}\right\|_{\infty}$ & 4.6858 & $2.8210^{5}$ \\
\hline
\end{tabular}

of the) observer are located at $(-1)$. The same value of $\theta(=150)$ has also been used.

The estimates of the system states provided by the NPFHGO are given in Fig. 3 where they are compared to their true (noise-free) values issued from the model simulation. The smooth state estimates provided by the observer using noisy output measurements as well as the absence of peaking of these estimates are worth to be emphasized.

For comparison purposes, the estimates provided by the SHGO have been reported in Fig. 4 where they are compared to their true (noise-free) values issued from the model simulation. The obtained estimates do highlight the peaking phenomenon of the SHGO during the transient periods as well as its sensitivity to the measurement noise. This fact is also emphasized by Table 1 where the infinity norm of each component of the observation error related to the state variables $x_{i}$ and denoted by $\left\|\tilde{x}_{i}\right\|_{\infty}, i=1, \ldots, 4$ provided by both observers are reported.

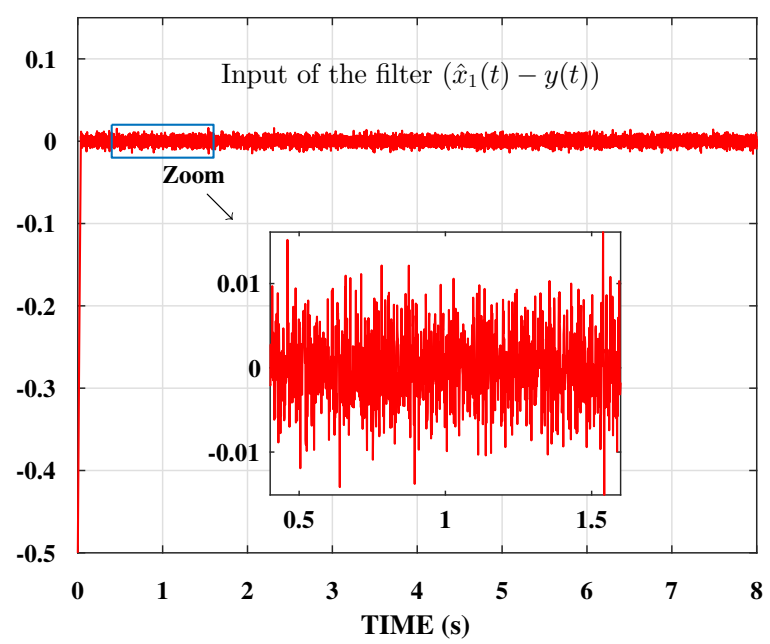

Fig. 5 Input and output signals of the filter in the NPFHGO
In order to put forward the filtering capabilities of the NPFHGO, the input and output signals of this filter are given in Fig. 5. Recall that the input of this filter is the noisy output observation error and its output is the filtered version $\eta_{1}(t)$ which is used by the corrective term of the observer [see (52)].

\section{Conclusion}

A SHGO redesign has been considered bearing in mind its two main known limitations, namely the measurement noise sensitivity and the peaking phenomenon occurring during the transient periods, leading thereby to a NPFHGO. The main properties of the NPFHGO have been highlighted and compared to those of its underlying SHGO through promising simulation results. The proposed observer redesign has been performed for a class of uniformly observable systems assuming that the output measurements are continuously available. Further studies to extend the proposed redesign to the case of sampled output measurements and to the class of non uniformly observable systems are under consideration.

Author contributions The main contributions are summarized in the Abstract.

Funding Research supported in part by the "Agence Nationale de la Recherche" (ANR), Project No. ANR-19-CE10-0007-04, France.

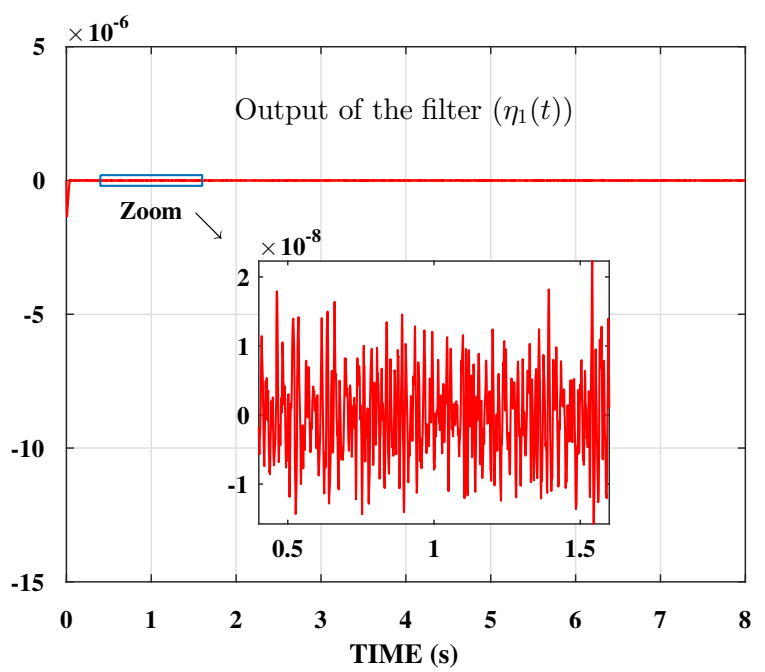


Open Access This article is licensed under a Creative Commons Attribution 4.0 International License, which permits use, sharing, adaptation, distribution and reproduction in any medium or format, as long as you give appropriate credit to the original author(s) and the source, provide a link to the Creative Commons licence, and indicate if changes were made. The images or other third party material in this article are included in the article's Creative Commons licence, unless indicated otherwise in a credit line to the material. If material is not included in the article's Creative Commons licence and your intended use is not permitted by statutory regulation or exceeds the permitted use, you will need to obtain permission directly from the copyright holder. To view a copy of this licence, visit http://creativecommons.org/licenses/ by/4.0/.

\section{A Proof of Proposition 3.1}

From (23) and (30), one has

$$
\begin{aligned}
\dot{\tilde{x}}= & A_{n} \tilde{x}+\tilde{\varphi}^{s}(u, \hat{x}, x) \\
& -\theta H\left(\tilde{x}_{1}\right)-B_{n, n} \varepsilon(t)-B_{n, i_{w}} w(t),
\end{aligned}
$$

where $\tilde{\varphi}^{s}(u, \hat{x}, x)=\varphi^{s}(u, \hat{x})-\varphi^{s}(u, x)$.

Since $\left|\tilde{x}_{1}\left(t_{0}\right)\right| \in \mathcal{S}_{-1}$, one has $\left|\gamma_{1} \tilde{x}_{1}\left(t_{0}\right)\right|>v$. Moreover and from the continuity of $\left|\tilde{x}_{1}(t)\right|$ with respect to time, there exists $t>t_{0}$ such that $\forall s \in$ $\left[t_{0}, t\right], \quad\left|\gamma_{1} \tilde{x}_{1}(s)\right|>v$. It is clear that $\tilde{x}_{1}(s)$ keeps the same sign for all $s \in\left[t_{0}, t\right]$. Moreover, as long as $\left|\gamma_{1} \tilde{x}_{1}(t)\right|>v$ and according to (32), one has $H\left(\tilde{x}_{1}(t)\right)=\operatorname{vsign}\left(\tilde{x}_{1}(t)\right) U_{n}$.

Using the comparison lemma, Eq. (55) leads to

$$
\begin{aligned}
\tilde{x}(t)= & e^{A_{n}\left(t-t_{0}\right)} \tilde{x}\left(t_{0}\right)+\int_{t_{0}}^{t} e^{A_{n}(t-s)} \tilde{\varphi}^{s}(u, \hat{x}, x) \mathrm{d} s \\
& -v \theta \operatorname{sign}\left(\tilde{x}_{1}(t)\right) \int_{t_{0}}^{t} e^{A_{n}(t-s)} U_{n} \mathrm{~d} s \\
& -\int_{t_{0}}^{t} e^{A_{n}(t-s)} B_{n, n} \varepsilon(s) \mathrm{d} s \\
& -\int_{t_{0}}^{t} e^{A_{n}(t-s)} B_{n, i_{w}} w(s) \mathrm{d} s,
\end{aligned}
$$

where $U_{n}^{T}=\left[\begin{array}{lll}1 & \ldots & 1\end{array}\right] \in \mathbb{R}^{n}$ defined as in (5).

The first component $\tilde{x}_{1}$ can be expressed as follows

$$
\begin{aligned}
\tilde{x}_{1}(t)= & \tilde{x}_{1}\left(t_{0}\right)+\sum_{k=1}^{n-1}\left(\frac{\left(t-t_{0}\right)^{k}}{k !} \tilde{x}_{k+1}\left(t_{0}\right)\right) \\
& +\sum_{k=1}^{n}\left(\int_{t_{0}}^{t} \frac{(t-s)^{k-1}}{(k-1) !} \tilde{\varphi}_{k}^{s}(u, \hat{x}, x) d s\right) \\
& -\int_{t_{0}}^{t} \frac{(t-s)^{i_{w}-1}}{\left(i_{w}-1\right) !} w(s) d s
\end{aligned}
$$

$$
\begin{aligned}
& -\int_{t_{0}}^{t} \frac{(t-s)^{n-1}}{(n-1) !} \varepsilon(s) d s \\
& -\nu \theta \operatorname{sign}\left(\tilde{x}_{1}(t)\right) \sum_{k=1}^{n} \frac{\left(t-t_{0}\right)^{k}}{k !} .
\end{aligned}
$$

Multiplying both sides of the above equality by $\operatorname{sign}\left(\tilde{x}_{1}(t)\right)\left(=\operatorname{sign}\left(\tilde{x}_{1}\left(t_{0}\right)\right)\right)$ and bounding its left side lead to

$$
\begin{aligned}
\left|\tilde{x}_{1}(t)\right| \leq & \left|\tilde{x}_{1}\left(t_{0}\right)\right|+\left\|\tilde{x}\left(t_{0}\right)\right\| \sum_{k=1}^{n-1} \frac{\left(t-t_{0}\right)^{k}}{k !} \\
& +\sum_{k=1}^{n} \int_{t_{0}}^{t} \frac{(t-s)^{k-1}}{(k-1) !}\left|\tilde{\varphi}_{k}^{s}(u, \hat{x}, x)\right| \mathrm{d} s \\
& +\int_{t_{0}}^{t} \frac{(t-s)^{i_{w}-1}}{\left(i_{w}-1\right) !}|w(s)| \mathrm{d} s \\
& +\int_{t_{0}}^{t} \frac{(t-s)^{n-1}}{(n-1) !}|\varepsilon(s)| \mathrm{d} s \\
& -v \theta \sum_{k=1}^{n} \frac{\left(t-t_{0}\right)^{k}}{k !} .
\end{aligned}
$$

One shall now respectively derive a bound for the third, fourth and fifth terms of the right side of the above inequality. Indeed, according to (18), one has

$$
\begin{aligned}
& \sum_{k=1}^{n} \int_{t_{0}}^{t}\left(\frac{(t-s)^{k-1}}{(k-1) !}\left|\tilde{\varphi}_{k}^{s}(u, \hat{x}, x)\right| \mathrm{d} s\right) \\
& \quad \leq 2 n L_{\varphi} R \sum_{k=1}^{n} \frac{\left(t-t_{0}\right)^{k}}{k !} .
\end{aligned}
$$

Similarly, according to (12) and (13), one has

$$
\begin{gathered}
\int_{t_{0}}^{t} \frac{(t-s)^{n-1}}{(n-1) !}|\varepsilon(s)| \mathrm{d} s \leq \frac{\left(t-t_{0}\right)^{n}}{n !} \delta_{\varepsilon} \text { and } \\
\int_{t_{0}}^{t} \frac{(t-s)^{i_{w}-1}}{\left(i_{w}-1\right) !}|w(s)| \mathrm{d} s \leq \frac{\left(t-t_{0}\right)^{i_{w}}}{i_{w} !} \delta_{w} .
\end{gathered}
$$

Using (58) and (59), inequality (57) leads to

$$
\begin{aligned}
\left|\tilde{x}_{1}(t)\right| \leq & \left|\tilde{x}_{1}\left(t_{0}\right)\right|+\left(\delta_{-1,0}+\delta_{w}\right. \\
& \left.+2 n L_{\varphi} R-v \theta\right) \sum_{k=1}^{n} \frac{\tau^{k}}{k !},
\end{aligned}
$$

where $\tau=t-t_{0}$ and $\delta_{-1,0}=\max \left(\left\|\tilde{x}\left(t_{0}\right)\right\|, \delta_{\varepsilon}\right)$.

One can check that for

$\theta>\theta_{-1,0}^{(1)} \triangleq \frac{2}{v}\left(\delta_{-1,0}+\delta_{w}+2 n L_{\varphi} R\right)$,

and inequality (60) leads to

$$
\left|\tilde{x}_{1}(t)\right| \leq\left|\tilde{x}_{1}\left(t_{0}\right)\right|-\frac{\nu \theta}{2} \sum_{k=1}^{n} \frac{\left(t-t_{0}\right)^{k}}{k !}
$$




$$
\leq\left|\tilde{x}_{1}\left(t_{0}\right)\right|-\frac{\nu \theta}{2} \tau
$$

Now, set

$$
\tau_{-1,0}^{(\star)}=\frac{\left|\tilde{x}_{1}\left(t_{0}\right)\right|-\frac{\nu}{\gamma_{1}}}{\frac{\nu \theta}{2}} .
$$

Notice that one has $\tau_{-1,0}^{(\star)}>0$ since $\left|\tilde{x}_{1}\left(t_{0}\right)\right| \in \mathcal{S}_{-1}$. Let $t_{-1,0}^{\star}=t_{0}+\tau_{-1,0}^{\star}$. According to (62), one gets for $t=t_{-1,0}^{\star}$,

$$
\left|\tilde{x}_{1}\left(t_{-1,0}^{\star}\right)\right| \leq\left|\tilde{x}_{1}\left(t_{0}\right)\right|-\frac{\nu \theta}{2} \tau_{-1,0}^{(\star)}=\frac{\nu}{\gamma_{1}} .
$$

According to (64), $\left|\tilde{x}_{1}(t)\right|$ will enter $\mathcal{S}_{0}$ after having evolved in the sector $\mathcal{S}_{-1}$ during a time period that does not exceed $\tau_{-1,0}^{(\star)}$. Hence, $t_{-1,0}^{(1)}$ exists and one has $\tau_{-1,0}^{(1)} \triangleq t_{-1,0}^{(1)}-t_{0} \leq \tau_{-1,0}^{(\star)}$. Now, from (63), one has $\lim _{\theta \rightarrow \infty} \tau_{-1,0}^{(\star)}=0$ which implies that $\lim _{\theta \rightarrow \infty} \tau_{-1,0}^{(1)}=0$ and hence item (i) of the proposition is proved.

Let us now look for $\alpha_{-1,0, \theta}^{(1)}$ required by item (ii). Indeed, using (56), (12), (13) and (22), one gets for all $t_{0} \leq t \leq t_{-1,0}^{(1)}$,

$$
\begin{aligned}
\|\tilde{x}(t)\| \leq & \left\|e^{A_{n}\left(t-t_{0}\right)}\right\|\left\|\tilde{x}\left(t_{0}\right)\right\| \\
& +\left(2 n \sqrt{n} L_{\varphi} R+v \sqrt{n} \theta+\delta_{w}+\delta_{\varepsilon}\right) \\
& \int_{t_{0}}^{t}\left\|e^{A_{n}(t-s)}\right\| \mathrm{d} s \\
\leq & \left\|\tilde{x}\left(t_{0}\right)\right\| \sum_{k=0}^{n-1}\left\|A_{n}^{k}\right\| \frac{\left(t-t_{0}\right)^{k}}{k !} \\
& +\left(2 n \sqrt{n} L_{\varphi} R+v \sqrt{n} \theta+\delta_{w}+\delta_{\varepsilon}\right) \\
& \int_{t_{0}}^{t} \sum_{k=1}^{n}\left\|A_{n}^{k-1}\right\| \frac{(t-s)^{k-1}}{(k-1) !} \mathrm{d} s \\
\leq & 2 \sqrt{n} R \sum_{k=0}^{n-1} \frac{\left(t-t_{0}\right)^{k}}{k !} \\
& +\left(2 n \sqrt{n} L_{\varphi} R+v \sqrt{n} \theta+\delta_{w}+\delta_{\varepsilon}\right) \\
& \sum_{k=1}^{n} \frac{\left(t-t_{0}\right)^{k}}{k !} \\
\leq & 2 \sqrt{n} R+\left(2 \sqrt{n}\left(n L_{\varphi}+1\right) R\right. \\
& \left.+v \sqrt{n} \theta+\delta_{w}+\delta_{\varepsilon}\right) \\
& \sum_{k=1}^{n}\left(t_{-1,0}^{(1)}-t_{0}\right)^{k} \triangleq \alpha_{-1,0, \theta}^{(1)} .
\end{aligned}
$$

Using the fact that $\lim _{\theta \rightarrow \infty}\left(t_{-1,0}^{(1)}-t_{0}\right)=0$, one can deduce from the expression of $\alpha_{-1,0, \theta}^{(1)}$ given by (65) that $\lim _{\theta \rightarrow \infty}\left(\alpha_{-1,0, \theta}^{(1)} / \theta\right)=0$. This ends the proof of Proposition 3.1.

\section{B Proof of Proposition 3.2}

One shall prove the Proposition by induction on $k$. Indeed, for $k=0$, the result is provided by Proposition 3.1. Now, let $k$ be fixed in $\{1, \ldots, n-1\}$ and assume that items (i) and (ii) of Proposition 3.2 hold for $k-1$, i.e.

(i) $t_{k-2, k-1}^{(1)}>0$ exists with

$$
\lim _{\theta \rightarrow \infty}\left(t_{k-2, k-1}^{(1)}-t_{0}\right)=0
$$

(ii) $\exists \alpha_{k-2, k-1, \theta}^{(1)}>0 ; \quad \forall t \leq t_{k-2, k-1}^{(1)}$,

$$
\begin{gathered}
\|\tilde{x}(t)\| \leq \alpha_{k-2, k-1, \theta}^{(1)} \text { with } \\
\lim _{\theta \rightarrow \infty}\left(\alpha_{k-2, k-1, \theta}^{(1)} / \theta\right)=0 .
\end{gathered}
$$

Let $t>t_{k-2, k-1}^{(1)}$ such that $\forall s \in\left[t_{k-2, k-1}^{(1)}, t\right],\left|\tilde{x}_{1}(s)\right| \in$ $\mathcal{S}_{k-1}$. Recall that since $\left|\tilde{x}_{1}(s)\right| \in \mathcal{S}_{k-1}$, then according to (35) and (34), the components of $H\left(\tilde{x}_{1}(s)\right)$ can be expressed as follows for all $s \in\left[t_{k-2, k-1}^{(1)}, t\right]$,

$$
\begin{cases}H_{j}\left(\tilde{x}_{1}(s)\right)=\gamma_{j} \theta^{j-1} \tilde{x}_{1}(s) & \text { for } j=1, \ldots, k, \\ H_{j}\left(\tilde{x}_{1}(s)\right)=v \operatorname{sign}\left(\tilde{x}_{1}(s)\right) & \text { for } j=k+1, \ldots, n .\end{cases}
$$

Using the comparison lemma, the solution of the ODE (55) can be expressed as follows

$$
\begin{aligned}
\tilde{x}(t)= & e^{A_{n}\left(t-t_{k-2, k-1}^{(1)}\right)} \tilde{x}\left(t_{k-2, k-1}^{(1)}\right) \\
& +\int_{t_{k-2, k-1}^{(1)}}^{t} e^{A_{n}(t-s)} \tilde{\varphi}^{s}(u, \hat{x}, x) \mathrm{d} s \\
& -\theta \int_{t_{k-2, k-1}^{(1)}}^{t} e^{A_{n}(t-s)} H\left(\tilde{x}_{1}(s)\right) \mathrm{d} s \\
& -\int_{t_{k-2, k-1}^{(1)}}^{t} e^{A_{n}(t-s)} B_{n, n} \varepsilon(s) \mathrm{d} s \\
& -\int_{t_{k-2, k-1}^{(1)}}^{t} e^{A_{n}(t-s)} B_{n, i_{w}} w(s)(s) \mathrm{d} s .
\end{aligned}
$$

Proceeding as in the proof of Proposition 3.1, i.e. substituting the components of $H\left(\tilde{x}_{1}(t)\right)$ by their expressions given by (67) and taking into account the fact that $\operatorname{sign}\left(\tilde{x}_{1}(t)\right)=\operatorname{sign}\left(\tilde{x}_{1}(s)\right)$ for all $s \in\left[t_{k-2, k-1}^{(1)}, t\right]$, the output observation error $\left|\tilde{x}_{1}(t)\right|$ can be bounded as follows

$\left|\tilde{x}_{1}(t)\right| \leq \sum_{j=0}^{n-1}\left(\frac{\left(t-t_{k-2, k-1}^{(1)}\right)^{j}}{j !}\left|\tilde{x}_{j+1}\left(t_{k-2, k-1}^{(1)}\right)\right|\right)$ 


$$
\begin{aligned}
& +\sum_{j=1}^{n} \int_{t_{k-2, k-1}^{(1)}}^{t}\left(\frac{(t-s)^{j-1}}{(j-1) !}\left|\tilde{\varphi}_{j}^{s}(u, \hat{x}, x)\right| \mathrm{d} s\right) \\
& -\theta\left(\sum_{j=1}^{k} \int_{t_{k-2, k-1}^{(1)}}^{t} \theta^{j-1} \gamma_{j} \frac{(t-s)^{j-1}}{(j-1) !}\left|\tilde{x}_{1}(s)\right| \mathrm{d} s\right. \\
& \left.+v \sum_{j=k+1}^{n} \int_{t_{k-2, k-1}^{(1)}}^{t} \frac{(t-s)^{j-1}}{(j-1) !} \mathrm{d} s\right) \\
& +\int_{t_{k-2, k-1}^{(1)}}^{t} \frac{(t-s)^{n-1}}{(n-1) !}|\varepsilon(s)| \mathrm{d} s \\
& +\int_{t_{k-2, k-1}^{(1)}}^{t} \frac{(t-s)^{i_{w}-1}}{\left(i_{w}-1\right) !}|w(s)| \mathrm{d} s .
\end{aligned}
$$

Note that according to the induction assumption, one has

$$
\left\|\tilde{x}\left(t_{k-2, k-1}^{(1)}\right)\right\| \leq \alpha_{k-2, k-1, \theta}^{(1)} .
$$

Using (70), the Lipschitz property (18) and the essential bounds provided by (12) and (13), inequality (69) leads to

$$
\begin{aligned}
\left|\tilde{x}_{1}(t)\right| \leq & \left|\tilde{x}_{1}\left(t_{k-2, k-1}^{(1)}\right)\right| \\
& +\alpha_{k-2, k-1, \theta}^{(1)} \sum_{j=1}^{n-1} \frac{\left(t-t_{k-2, k-1}^{(1)}\right)^{j}}{j !} \\
& +2 n L_{\varphi} R \sum_{j=1}^{n} \frac{\left(t-t_{k-2, k-1}^{(1)}\right)^{j}}{j !} \\
& -\theta v \sum_{j=k+1}^{n} \frac{\left(t-t_{k-2, k-1}^{(1)}\right)^{j}}{j !} \\
& +\delta_{\varepsilon} \frac{\left(t-t_{k-2, k-1}^{(1)}\right)^{n}+\delta_{w} \frac{\left(t-t_{k-2, k-1}^{(1)}\right)^{i_{w}}}{n !}}{\leq} \\
& \left|\tilde{x}_{1}\left(t_{k-2, k-1}^{(1)}\right)\right|+\left(\delta_{k-1, k, \theta}^{(1)}\right. \\
& \left.+\delta_{w}+2 n L_{\varphi} R\right) \sum_{j=1}^{n} \frac{\left(t-t_{k-2, k-1}^{(1)}\right)^{j}}{j !} \\
& -\theta v \sum_{j=k+1}^{n} \frac{\left(t-t_{k-2, k-1}^{(1)}\right)^{j}}{j !} \\
& +71)
\end{aligned}
$$

where $\delta_{k-1, k, \theta}^{(1)}=\max \left(\alpha_{k-2, k-1, \theta}^{(1)}, \delta_{\varepsilon}\right)$. Now, set

$\bar{\delta}_{k-1, k, \theta}^{(1)}=\delta_{k-1, k, \theta}^{(1)}+\delta_{w}+2 n L_{\varphi} R$.

Notice that since $\lim _{\theta \rightarrow \infty}\left(\alpha_{k-2, k-1, \theta}^{(1)} / \theta\right)=0$, one has $\lim _{\theta \rightarrow \infty}\left(\delta_{k-2, k-1, \theta}^{(1)} / \theta\right)=\lim _{\theta \rightarrow \infty}\left(\bar{\delta}_{k-2, k-1, \theta}^{(1)}\right.$ $(\theta)=0$ and as a result there exists $\theta_{k-1, k}^{(1)}>0$ such that

$\forall \theta \geq \theta_{k-1, k}^{(1)}, \quad \bar{\delta}_{k-1, k, \theta}^{(1)} \leq \frac{\nu \theta}{2}$.

Combining (71)-(73), one gets

$$
\begin{aligned}
\left|\tilde{x}_{1}(t)\right| \leq & \left|\tilde{x}_{1}\left(t_{k-2, k-1}^{(1)}\right)\right|+\bar{\delta}_{k-1, k, \theta}^{(1)} \sum_{j=1}^{k} \frac{\left(t-t_{k-2, k-1}^{(1)}\right)^{j}}{j !} \\
& -\frac{\theta v}{2} \sum_{j=k+1}^{n} \frac{\left(t-t_{k-2, k-1}^{(1)}\right)^{j}}{j !} \\
\leq & \left|\tilde{x}_{1}\left(t_{k-2, k-1}^{(1)}\right)\right|+\bar{\delta}_{k-1, k, \theta}^{(1)} \sum_{j=1}^{k} \frac{\left(t-t_{k-2, k-1}^{(1)}\right)^{j}}{j !} \\
& -\frac{\theta v}{2} \frac{\left(t-t_{k-2, k-1}^{(1)}\right)^{k+1}}{(k+1) !} \\
\triangleq & P_{k-1, k}^{(1)}(\tau)+\frac{v}{\gamma_{k+1} \theta^{k}}
\end{aligned}
$$

where $\tau=t-t_{k-2, k-1}^{(1)}$ and

$$
\begin{aligned}
& P_{k-1, k}^{(1)}(\tau) \triangleq a_{0, k-1, k}^{(i)}+\sum_{j=1}^{k} a_{j, k-1, k}^{(1)} \tau^{j} \\
& -\theta a_{k+1, k-1, k}^{(1)} \tau^{k+1}, \\
& \text { with }\left\{\begin{array}{r}
a_{0, k-1, k}^{(1)}=\left(\left|\tilde{x}_{1}\left(t_{k-2, k-1}^{(1)}\right)\right|-\frac{v}{\gamma_{k+1} \theta^{k}}\right) \\
=\left(\frac{v}{\gamma_{k} \theta^{k-1}}-\frac{v}{\gamma_{k+1} \theta^{k}}\right), \\
\bar{\delta}_{j, k-1, k}^{(1)} \\
a_{k-1, k, \theta}^{(1)}, j=1, \ldots, k \text { and } \\
a_{k+1, k-1, k}^{(1)}=\frac{v}{2(k+1) !} .
\end{array}\right.
\end{aligned}
$$

According to inequality (76), it is guaranteed that $\left|\tilde{x}_{1}(t)\right|$ is lower than or equal to $\frac{v}{\gamma_{k+1} \theta^{k}}$ if $P_{k-1, k}^{(1)}(\tau) \leq 0$. Otherwise said, if $P_{k-1, k}^{(1)}(\tau) \leq 0$, then it is guaranteed that $\left|\tilde{x}_{1}(t)\right|$ has already left $\mathcal{S}_{k-1}$ and entered $\mathcal{S}_{k}$. Hence, one shall focus in what follows on the sign of $P_{k-1, k}^{(1)}(\tau)$.

It is clear from (78) that the $a_{j, k-1, k}^{(1)}$ 's, $j=0, \ldots, n$ are positive. Hence, according to Descartes' rule of signs [29], the number of positive roots of the polynomial $P_{k-1, k}^{(1)}(\tau)$ is equal to 1 . This means that there exists a unique real $\tau_{k-1, k}^{\star}>0$, such that $P_{k-1, k}^{(1)}\left(\tau_{k-1, k}^{\star}\right)=0$. Since $P_{k-1, k}^{(1)}(0)=a_{0, k-1, k}^{(1)}>0$, one has for all $\tau \geq \tau_{k-1, k}^{\star}, P_{k-1, k}^{(1)}(\tau) \leq 0$.

Notice that inequality (76) is valid as long as $\left|\tilde{x}_{1}(t)\right|$ evolves in the sector $\mathcal{S}_{k-1}$ and it cannot come back to $\mathcal{S}_{k-2}$ before the changing of its sign. Otherwise said, $\left|\tilde{x}_{1}(t)\right|$ will evolve in $\mathcal{S}_{k-1}$ since the time instant $t_{k-2, k-1}^{(1)}$ and it will leave it to enter $\mathcal{S}_{k}$ at a some time 
instant which is denoted $t_{k-1, k}^{(1)}$ according to the adopted notation. From (76), the time period of the staying of $\left|\tilde{x}_{1}(t)\right|$ in $\mathcal{S}_{k-1}$ cannot exceed $\tau_{k-1, k}^{\star}$, i.e.

$\tau_{k-1, k}^{(1)} \triangleq t_{k-1, k}^{(1)}-t_{k-2, k-1}^{(1)} \leq \tau_{k-1, k}^{\star}$.

Let us now calculate the limit of $\tau_{k-1, k}^{(1)}$ when $\theta$ goes to $\infty$.

Since $\tau_{k-1, k}^{\star}$ is a root of $P_{k-1, k}^{(1)}(\tau)=0$ and according to (77), it satisfies the following equality,

$$
\begin{aligned}
& \frac{a_{0, k-1, k, \theta}^{(1)}}{\theta}+\sum_{j=1}^{k} \frac{a_{j, k-1, k, \theta}^{(1)}}{\theta}\left(\tau_{k-1, k}^{\star}\right)^{j} \\
& -a_{k+1, k-1, k}^{(1)}\left(\tau_{k-1, k}^{\star}\right)^{k+1}=0 .
\end{aligned}
$$

One clearly has $\lim _{\theta \rightarrow \infty}\left(a_{j, k-1, k, \theta}^{(1)} / \theta\right)=0, j=$ $0, \ldots, k$. Using this fact, Eq. (80) leads to

$\lim _{\theta \rightarrow \infty}\left(a_{k+1, k-1, k}^{(1)}\left(\tau_{k-1, k}^{\star}\right)^{k+1}\right)=0$.

Since $a_{k+1, k-1, k}^{(1)}=\frac{v}{2(k+1) !}$, the above equality implies that $\lim _{\theta \rightarrow \infty} \tau_{k-1, k}^{\star}=0$ and according to (79), one gets $\lim _{\theta \rightarrow \infty} \tau_{k-1, k}^{(1)}=0$. This leads to

$$
\begin{aligned}
& \lim _{\theta \rightarrow \infty}\left(t_{k-1, k}^{(1)}-t_{0}\right) \\
& =\lim _{\theta \rightarrow \infty}\left(t_{k-1, k}^{(1)}-t_{k-2, k-1}^{(1)}+t_{k-2, k-1}^{(1)}-t_{0}\right) \\
& =\lim _{\theta \rightarrow \infty}\left(\tau_{k-1, k}^{(1)}+t_{k-2, k-1}^{(1)}-t_{0}\right) \\
& =\lim _{\theta \rightarrow \infty} \tau_{k-1, k}^{(1)} \text { according to (66) } \\
& =0 .
\end{aligned}
$$

Let us now look for the positive real $\alpha_{k-1, k, \theta}^{(1)}$ of item (ii) in Proposition 3.2. Indeed, proceeding as in the proof of Proposition 3.1, one gets for all $t_{k-2, k-1}^{(1)} \leq$ $t \leq t_{k-1, k}^{(1)}$, [compare with (65)],

$$
\begin{aligned}
\|\tilde{x}(t)\| \leq & \left\|\tilde{x}\left(t_{k-2, k-1}^{(1)}\right)\right\| \\
& \left(\left\|\tilde{x}\left(t_{k-2, k-1}^{(1)}\right)\right\|+2 n \sqrt{n} L_{\varphi} R\right. \\
& \left.+v \sqrt{n} \theta+\delta_{w}+\delta_{\varepsilon}\right) \\
& \sum_{j=1}^{n}\left(t_{k-1, k}^{(1)}-t_{k-2, k-1}^{(1)}\right)^{j} \\
\leq & \alpha_{k-2, k-1, \theta}^{(1)}+\left(\alpha_{k-2, k-1, \theta}^{(1)}+2 n \sqrt{n} L_{\varphi} R\right. \\
& \left.+v \sqrt{n} \theta+\delta_{w}+\delta_{\varepsilon}\right) \sum_{j=1}^{n}\left(\tau_{k-1, k}^{(1)}\right)^{j}
\end{aligned}
$$

$$
\triangleq \alpha_{k-1, k, \theta}^{(1)} \text {. }
$$

From the fact that $\lim _{\theta \rightarrow \infty}\left(\alpha_{k-2, k-1, \theta}^{(1)} / \theta\right)=$ $\lim _{\theta \rightarrow \infty}$

$\tau_{k-1, k}^{(1)}=0$, one deduces from the expression of $\alpha_{k-1, k, \theta}^{(1)}$ given by (81) that $\lim _{\theta \rightarrow \infty}\left(\alpha_{k-1, k, \theta}^{(1)} / \theta\right)=0$. This ends the proof of Proposition 3.2.

\section{Proof of Proposition 3.3}

Let $t>t_{n-2, n-1}^{(1)}$ such that $\left|\tilde{x}_{1}(t)\right| \in \mathcal{S}_{n-1}$, i.e. $\left|\tilde{x}_{1}(t)\right| \leq \frac{v}{\gamma_{n} \theta^{n-1}}$. According to (33), $H\left(\tilde{x}_{1}(t)\right)=$ $\Delta_{n}^{-1}(\theta) \Gamma_{n} \tilde{x}_{1}(t)$. Moreover, since the essential bound $\delta_{w}$ satisfies (28), then each component of the observation error satisfies an inequality similar to (29) which specializes as follows when accounting for (36),

$$
\begin{aligned}
\left|\underline{\tilde{x}}_{i}(t)\right| \leq & \sigma_{P_{n}}\left(\theta^{i-1} e^{-\beta_{n} \theta\left(t-t_{n-2, n-1}^{(1)}\right)} \alpha_{n-2, n-1, \theta}^{(1)}\right. \\
& \left.+\frac{\delta_{\varepsilon}+\bar{\delta}_{w}}{\theta^{n+1-i} \beta_{n}}\right), \quad i=1, \ldots, n .
\end{aligned}
$$

In particular,

$$
\begin{aligned}
\left|\tilde{x}_{1}(t)\right|= & \left|\underline{\tilde{x}}_{1}(t)\right| \leq \sigma_{P_{n}}\left(e^{-\theta \beta_{n}\left(t-t_{n-2, n-1}^{(1)}\right)} \alpha_{n-2, n-1, \theta}^{(1)}\right. \\
& \left.+\frac{\delta_{\varepsilon}+\bar{\delta}_{w}}{\theta^{n} \beta_{n}}\right)
\end{aligned}
$$

If the sequence $\left\{t_{n-1, n-2}^{(i)}\right\}_{i \geq 1}$ is non empty, then $\left|\tilde{x}_{1}(t)\right|$ leaves $\mathcal{S}_{n-1}$ and enters $\mathcal{S}_{n-2}$ at the time instant $t_{n-1, n-2}^{(1)}$. Using (83), one gets for $t=t_{n-1, n-2}^{(1)}$,

$$
\begin{aligned}
\left|\tilde{x}_{1}\left(t_{n-1, n-2}^{(1)+}\right)\right| & =\frac{v}{\gamma_{n} \theta^{n-1}} \\
& =\sigma_{P_{n}}\left(\alpha_{n-2, n-1, \theta}^{(1)} e^{-\theta \beta_{n} \tau_{n-1}^{(1)}}+\frac{\delta_{\varepsilon}+\bar{\delta}_{w}}{\theta^{n} \beta_{n}}\right) .
\end{aligned}
$$

One can check that the above equality leads to

$\tau_{n-1}^{(1)}=\frac{1}{\theta \beta_{n}} \log \left(\frac{\beta_{n} \sigma_{P_{n}} \alpha_{n-2, n-1, \theta}^{(1)} \gamma_{n} \theta^{n}}{\theta \nu \beta_{n}-\sigma_{P_{n}} \gamma_{n}\left(\delta_{\varepsilon}+\bar{\delta}_{w}\right)}\right)$.

It is clear from (84) that

$\lim _{\theta \rightarrow \infty} \tau_{n-1}^{(1)}=0$.

Let us now bound $\|\tilde{x}(t)\|$ for $t \leq t_{n-1, n-2}^{(1)}$. Indeed, as in the proofs of Propositions 3.1 and 3.2, one can show that for all $t \leq t_{n-1, n-2}^{(1)}$, [compare with (65) and (81)], $\|\tilde{x}(t)\| \leq\left\|\tilde{x}\left(t_{n-2, n-1}^{(1)}\right)\right\|+\left(\left\|\tilde{x}\left(t_{n-2, n-1}^{(1)}\right)\right\|\right.$ 


$$
\begin{gathered}
\left.+2 n \sqrt{n} L_{\varphi} R+v \sqrt{n} \theta+\delta_{w}+\delta_{\varepsilon}\right) \\
\sum_{k=1}^{n}\left(t_{n-1, n-2}^{(1)}-t_{n-2, n-1}^{(1)}\right)^{k} .
\end{gathered}
$$

From the fact that $\left\|\tilde{x}\left(t_{n-2, n-1}^{(1)}\right)\right\| \leq \alpha_{n-2, n-1, \theta}^{(1)}$, one gets

$$
\begin{aligned}
\|\tilde{x}(t)\| \leq & \alpha_{n-2, n-1, \theta}^{(1)}+\left(\alpha_{n-2, n-1, \theta}^{(1)}+2 n \sqrt{n} L_{\varphi} R\right. \\
& \left.+v \sqrt{n} \theta+\delta_{w}+\delta_{\varepsilon}\right) \sum_{k=1}^{n}\left(\tau_{n-1}^{(1)}\right)^{k} \triangleq \alpha_{n-1, \theta}^{(1)} .
\end{aligned}
$$

Since $\lim _{\theta \rightarrow \infty}\left(\alpha_{n-2, n-1, \theta}^{(1)} / \theta\right)=\lim _{\theta \rightarrow \infty} \tau_{n-1}^{(1)}=0$, one can easily deduce from the expression of $\alpha_{n-1, \theta}^{(1)}$ given by (86) that $\lim _{\theta \rightarrow \infty}\left(\alpha_{n-1, \theta}^{(1)} / \theta\right)=0$. This ends the proof of Proposition 3.3.

\section{Proof of Proposition 3.4}

Let $t>t_{n-1, n-2}^{(1)}$ such that $\left.\left.\forall s \in\right] t_{n-1, n-2}^{(1)}, t\right],\left|\tilde{x}_{1}(s)\right| \in$ $\mathcal{S}_{n-2}$. Proceeding as in the proof of Proposition 3.2, one can show that for $\theta$ high enough, one has [compare with inequality (75)]

$$
\begin{aligned}
\left|\tilde{x}_{1}(t)\right| \leq & \left|\tilde{x}_{1}\left(t_{n-1, n-2}^{(1)}\right)\right| \\
& +\bar{\delta}_{n-1, \theta}^{(1)} \sum_{j=1}^{n-1} \frac{\left(t-t_{n-1, n-2}^{(1)}\right)^{j}}{j !} \\
& -\frac{\theta v}{2} \frac{\left(t-t_{n-1, n-2}^{(1)}\right)^{n}}{n !},
\end{aligned}
$$

where $\bar{\delta}_{n-1, \theta}^{(1)}=\max \left(\alpha_{n-1, \theta}^{(1)}, \delta_{\varepsilon}\right)+\delta_{w}+2 n L_{\varphi} R$.

Set $\tau=t-t_{n-1, n-2}^{(1)}$; the last inequality becomes

$$
\begin{aligned}
\left|\tilde{x}_{1}(t)\right| & \leq\left|\tilde{x}_{1}\left(t_{n-1, n-2}^{(1)}\right)\right|+\bar{\delta}_{n-1, \theta}^{(1)} \sum_{j=1}^{n-1} \frac{\tau^{j}}{j !}-\frac{\theta v}{2} \frac{\tau^{n}}{n !} \\
& =\frac{v}{\gamma_{n} \theta^{n-1}}+\bar{\delta}_{n-1, \theta}^{(1)} \sum_{j=1}^{n-1} \frac{\tau^{j}}{j !}-\frac{\theta v}{2} \frac{\tau^{n}}{n !} .
\end{aligned}
$$

According to the definition of $\bar{\delta}_{n-1, \theta}^{(1)}$ and since $\lim _{\theta \rightarrow \infty}$ $\left(\alpha_{n-1, \theta}^{(1)} / \theta\right)=0$, one has $\lim _{\theta \rightarrow \infty}\left(\bar{\delta}_{n-1, \theta}^{(1)} / \theta\right)=0$.

Now, $\left|\tilde{x}_{1}(t)\right|$ will evolve according to one of two scenarios. The first scenario corresponds to the case where $\left|\tilde{x}_{1}(t)\right|$ leaves $\mathcal{S}_{n-2}$ and enters $\mathcal{S}_{n-3}$. The second one deals with the case where $\left|\tilde{x}_{1}(t)\right|$ does not leave the sector $\mathcal{S}_{n-2}$ and still evolve inside it. In order to analyze the time evolution of $\left|\tilde{x}_{1}(t)\right|$ and in particular to identify the scenario according to which it will evolve, one introduces the following polynomial in $\tau$

$$
\begin{aligned}
& P_{n-1, n-2}^{(1)}(\tau) \triangleq a_{0, n-1, n-2}^{(1)}+\sum_{j=1}^{n-1} a_{j, n-1, n-2}^{(1)} \tau^{j} \\
& \quad-\theta a_{n, n-1, n-2}^{(1)} \tau^{n},
\end{aligned}
$$

with

$a_{0, n-1, n-2}^{(1)}=\left(\frac{v}{\gamma_{n} \theta^{n-1}}-\frac{v}{\gamma_{n-1} \theta^{n-2}}\right)$,

$a_{j, n-1, n-2}^{(1)}=\frac{\bar{\delta}_{n-1, \theta}^{(1)}}{j !}, j=1, \ldots, n-1$, and

$a_{n, n-1, n-2}^{(1)}=\frac{v}{2 n !}$.

Inequality (87) can be rewritten as follows

$$
\left|\tilde{x}_{1}(t)\right| \leq P_{n-1, n-2}^{(1)}(\tau)+\frac{v}{\gamma_{n-1} \theta^{n-2}} .
$$

From the above inequality, one can deduce the following property

$\left|\tilde{x}_{1}(t)\right|>\frac{v}{\gamma_{n-1} \theta^{n-2}} \Longrightarrow P_{n-1, n-2}^{(1)}(\tau)>0$.

Property (91) means that if there exists a time instant $t$ at which $\left|\tilde{x}_{1}(t)\right|$ leaves $\mathcal{S}_{n-2}$ and enters $\mathcal{S}_{n-3}$, then this time instant $t$ is such that $P_{n-1, n-2}^{(1)}(\tau)>0$ where $\tau=t-t_{n-1, n-2}^{(1)}$. Hence, one shall focus in what follows on the sign of $P_{n-1, n-2}^{(1)}(\tau)$.

It is clear from (89) that $a_{0, n-1, n-2}^{(1)}<0$ and $a_{j, n-1, n-2}>0, j=1, \ldots, n$. Hence, the number of changes of the coefficient signs in the polynomial $P_{n-1, n-2}^{(1)}$ is equal to 2 and according to Descartes' rule of signs, the polynomial $P_{n-1, n-2}^{(1)}$ admits either 2 or zero positive real roots. Let us consider these two cases.

Case 1: $P_{n-1, n-2}^{(1)}(\tau)$ admits two positive real roots. Let denote by $\tau_{1}$ and $\tau_{2}$ these roots. Since $P_{n-1, n-2}^{(1)}(0)=$ $a_{0, n-1, n-2}^{(1)}<0$, one deduces that $\left.\forall \tau \in\right] \tau_{1}, \tau_{2}[$, $P_{n-1, n-2}^{(1)}(\tau)>0$ and $\forall \tau \notin\left[\tau_{1}, \tau_{2}\right], P_{n-1, n-2}^{(1)}(\tau) \leq 0$. Hence, if the time instant $t_{n-2, n-3}^{(1)}$ exists, then one necessarily has $\left.\tau_{n-2, n-3}^{(1)} \triangleq t_{n-2, n-3}^{(1)}-t_{n-1, n-2}^{(1)} \in\right] \tau_{1}, \tau_{2}[$. Moreover, using similar developments as in the proof of Proposition 3.2, one can show that the two positive real roots $\tau_{1}$ and $\tau_{2}$ are such that $\lim _{\theta \rightarrow \infty} \tau_{i}=0$, $i=1,2$ and this implies that $\lim _{\theta \rightarrow \infty} \tau_{n-2, n-3}^{(1)}=0$. 
One can also show that there exists $\alpha_{n-2, n-3, \theta}^{(1)}$ such that for all $t \leq t_{n-2, n-3}^{(1)},\|\tilde{x}(t)\| \leq \alpha_{n-2, n-3, \theta}^{(1)}$ with $\lim _{\theta \rightarrow \infty}\left(\alpha_{n-2, n-3, \theta}^{(1)} / \theta\right)=0$.

Case 2: $P_{n-1, n-2}^{(1)}(\tau)$ admits no root. In this case, $P_{n-1, n-2}^{(1)}(\tau)$ keeps the same sign for all $\tau$. Since $P_{n-1, n-2}^{(1)}(0)=a_{0, n-1, n-2}^{(1)}<0$, one has $\forall \tau \geq$ $0, P_{n-1, n-2}^{(1)}(\tau)<0$. Combining this fact with inequality (90) allows to deduce that $\left|\tilde{x}_{1}(t)\right|<\frac{v}{\gamma_{n-1} \theta^{n-2}}$. Otherwise said, $\left|\tilde{x}_{1}(t)\right|$ cannot enter $\mathcal{S}_{n-3}$ and still evolve in $\mathcal{S}_{n-2}$. Let us analyze the behaviour of $\left|\tilde{x}_{1}(t)\right|$ when evolving in $\mathcal{S}_{n-2}$. For this aim, set $\tau=t-t_{n-1, n-2}^{(1)}$ and consider the following polynomial in $\tau$

$$
\begin{aligned}
P_{n-2}^{(1)}(\tau)= & \left(\left|\tilde{x}_{1}\left(t_{n-1, n-2}^{(1)}\right)\right|-\frac{v}{\gamma_{n} \theta^{n-1}}\right) \\
& +\bar{\delta}_{n-1, \theta}^{(1)} \sum_{j=1}^{n-1} \frac{\tau^{j}}{j !}-\frac{\theta v}{2} \frac{\tau^{n}}{n !} \\
= & \bar{\delta}_{n-1, \theta}^{(1)} \sum_{j=1}^{n-1} \frac{\tau^{j}}{j !}-\frac{\theta v}{2} \frac{\tau^{n}}{n !} \\
& \triangleq \sum_{j=1}^{n-1} a_{j, n-1, n-2}^{(1)} \tau^{j}-\theta a_{n, n-1, n-2}^{(1)} \tau^{j},
\end{aligned}
$$

where the $a_{j, n-1, n-2}^{(1)}$ 's, $j=1, \ldots, n$ are defined by (89).

Now, inequality (87) can be rewritten as follows

$\left|\tilde{x}_{1}(t)\right| \leq P_{n-2}^{(1)}(\tau)+\frac{v}{\gamma_{n} \theta^{n-1}}$.

From the above inequality, one can deduce the following property

$P_{n-2}^{(1)}(\tau)<0 \Longrightarrow\left|\tilde{x}_{1}(t)\right|<\frac{v}{\gamma_{n} \theta^{n-1}}$.

According to inequality (94), $\left|\tilde{x}_{1}(t)\right|$ will necessarily enter $\mathcal{S}_{n-1}$ when $P_{n-2}^{(1)}(\tau)$ becomes negative and it still evolve in $\mathcal{S}_{n-1}$ as long as $P_{n-2}^{(1)}(\tau)<0$. Hence, let us focus on the sign of $P_{n-2}^{(1)}(\tau)$.

Again, using Descartes' rule of signs, it clear that the polynomial equation $P_{n-2}^{(1)}(\tau)=0$ has a unique positive real root denoted by $\tau_{n-2}^{\star}$. Note that $\tau_{1} \triangleq 0$ is also a real root of $P_{n-2}^{(1)}$. Hence, the polynomial $P_{n-2}^{(1)}(\tau)$ remains positive when $\tau$ lies between the two non negative real roots, i.e. $\tau \in] 0, \tau_{n-2}^{\star}[$ and it becomes negative as soon as $\tau>\tau_{n-2}^{\star}$. This means that $\left|\tilde{x}_{1}(t)\right|$ will leave $\mathcal{S}_{n-2}$ to come back to $\mathcal{S}_{n-1}$ at the latest at the time instant $t=t_{n-1, n-2}^{(1)}+\tau_{n-2}^{\star}$. According to the adopted definitions and since $\left|\tilde{x}_{1}(t)\right|$ will enter $\mathcal{S}_{n-1}$ for the second time at this time instant, one deduces that $t_{n-2, n-1}^{(2)}$ exists and one has $t_{n-2, n-1}^{(2)} \leq$ $t_{n-1, n-2}^{(1)}+\tau_{n-2}^{\star}$ or equivalently $\tau_{n-2}^{(1)} \triangleq t_{n-2, n-1}^{(2)}-$ $t_{n-1, n-2}^{(1)} \leq \tau_{n-2}^{\star}$. Again, by using similar developments as in the proof of Proposition 3.2, one can show that $\lim _{\theta \rightarrow \infty} \tau_{n-2}^{(1)}=0$ and that there exists $\alpha_{n-2, \theta}^{(1)}$ such that for all $t \leq t_{n-2, n-1}^{(1)},\|\tilde{x}(t)\| \leq \alpha_{n-2, \theta}^{(1)}$ with $\lim _{\theta \rightarrow \infty}\left(\alpha_{n-2, \theta}^{(1)} / \theta\right)=0$.

To summarize, in the above developments describing the behaviour of $\left|\tilde{x}_{1}(t)\right|$ when leaving $\mathcal{S}_{n-1}$, the following two exclusive scenarios have been put forward:

1. The first scenario consists in the case where $\left|\tilde{x}_{1}(t)\right|$ may leave $\mathcal{S}_{n-2}$ and enter $\mathcal{S}_{n-3}$ at a time instant $t_{n-2, n-3}^{(1)}=t_{n-1, n-2}^{(1)}+\tau_{n-2, n-3}^{(1)}$ with $\lim _{\theta \rightarrow \infty} \tau_{n-2, n-3}^{(1)}=0$. Moreover, there exists $\alpha_{n-2, n-3, \theta}^{(1)}$ such that for all $t \leq t_{n-2, n-3}^{(1)},\|\tilde{x}(t)\| \leq$ $\alpha_{n-2, n-3, \theta}^{(1)}$ with $\lim _{\theta \rightarrow \infty}\left(\alpha_{n-2, n-3}^{(1)} / \theta\right)=0$.

2. The second scenario corresponds to the case where $\left|\tilde{x}_{1}(t)\right|$ still evolve in $\mathcal{S}_{n-2}$ during a time period equal to $\tau_{n-2}^{(1)}$ and then comes back to $\mathcal{S}_{n-1}$ at the time instant $t_{n-2, n-1}^{(2)}$ with $\lim _{\theta \rightarrow \infty} \tau_{n-2}^{(1)}=0$. Moreover, there exists $\alpha_{n-2, \theta}^{(1)}$ such that for all $t \leq$ $t_{n-2, n-1}^{(1)},\|\tilde{x}(t)\| \leq \alpha_{n-2, \theta}^{(1)}$ with $\lim _{\theta \rightarrow \infty}\left(\alpha_{n-2, \theta}^{(1)}\right.$ $(\theta)=0$.

According to these two possible scenarios, the reals $t_{k, k+1}^{(2)}, \tau_{k}^{(1)}$ and $\alpha_{k, \theta}^{(1)}>0$ required by the Proposition are obtained when the second scenario is occurring and one has $k=n-2$. In the case where the first scenario occurs but not the second one, the same reasoning can be pursued to analyze the behaviour of $\left|\tilde{x}_{1}(t)\right|$ from the time instant when it leaves $\mathcal{S}_{n-2}$ and enters $\mathcal{S}_{n-3}$. It is easy to see that $\left|\tilde{x}_{1}(t)\right|$ will still evolve according the above two exclusive scenarios. As soon as the second scenario occurs inside a sector $k$, the parameters required by the Proposition are obtained. Note that $k$ is assumed to be in $\{1, \ldots, n-1\}$ in the statement of the Proposition. Now, one will show that if $\left|\tilde{x}_{1}(t)\right|$ reaches the sector $\mathcal{S}_{0}$, only the second scenario will occur. Indeed, assume that there exists a time instant $t_{a}>t_{-1,0}^{(1)}$ such that $\left|\tilde{x}_{1}(t)\right|$ leaves $\mathcal{S}_{0}$ and enters $\mathcal{S}_{-1}$ at the time instant $t_{a}$. According to the adopted notation, one has $\left|\tilde{x}_{1}\left(t_{a}\right)\right|=\frac{\nu}{\gamma_{1}}$ and 
$\left|\tilde{x}_{1}\left(t_{a}^{+}\right)\right|>\frac{\nu}{\gamma_{1}}$. Taking into account the continuity of $\left|\tilde{x}_{1}(t)\right|$, one has $\left.\left.\exists t>t_{a} ; \forall s \in\right] t_{a}, t\right],\left|\tilde{x}_{1}(s)\right| \in \mathcal{S}_{-1}$. This in particular means that

$\left|\tilde{x}_{1}(t)\right|>\frac{v}{\gamma_{1}}$.

Since $\left|\tilde{x}_{1}(s)\right|$ evolves in $\mathcal{S}_{-1}$ for all $\left.\left.s \in\right] t_{a}, t\right]$, one can process as in the proof of Proposition 3.1 to derive the following inequality [compare with (62)]

$\left|\tilde{x}_{1}(t)\right| \leq\left|\tilde{x}_{1}\left(t_{a}\right)\right|-\frac{\nu \theta}{2}\left(t-t_{a}\right)$.

Notice that the above inequality (96) is valid under assumptions similar to those adopted to generate inequality (62). In particular, the design parameter $\theta$ is assumed to be high enough and satisfies [compare with (61)]

$\theta>\frac{2}{v}\left(\max \left(\left\|\tilde{x}\left(t_{a}\right)\right\|, \delta_{\varepsilon}\right)+\delta_{w}+2 n L_{\varphi} R\right)$.

The choice of $\theta$ as in (97) is possible since $\left\|\tilde{x}\left(t_{a}\right)\right\| \leq \alpha_{\theta}$ with $\lim _{\theta \rightarrow \infty} \frac{\alpha_{\theta}}{\theta}=0$ (see (37)).

Combining (96) with the fact that $t>t_{a}$ leads to $\left|\tilde{x}_{1}(t)\right| \leq\left|\tilde{x}_{1}\left(t_{a}\right)\right|=\frac{v}{\gamma_{1}}$ which is in contradiction with inequality (95). Hence, $\left|\tilde{x}_{1}(t)\right|$ will evolve according to the second scenario inside the sector $\mathcal{S}_{0}$, i.e. it will leave it to enter $\mathcal{S}_{1}$. This ends the proof of Proposition 3.4.

\section{E Proof of Proposition 3.5}

Let $i_{k}$ denote the number of terms in the sequence $\left\{t_{k-1, k}^{(i)}\right\}_{i \geq 1}$ when this sequence is finite. The proof of the Proposition will be achieved by induction on $k$. Indeed, for $k=0$ one has $i_{0}=1$, i.e. $\mid \tilde{x}_{1}(t)$ never comes back to $\mathcal{S}_{-1}$ after the time instant $t_{-1,0}^{(1)}$ as detailed in the end of the proof of Proposition 3.4.

Now, for a fixed $k \in\{1, \ldots, n-1\}$, one shall assume that $\forall j \in\{1, \ldots, k-1\}$, the sequence $\left\{t_{j-1, j}^{(i)}\right\}_{i \geq 1}$ is finite and one has to prove that the sequence $\left\{t_{k-1, k}^{(i)}\right\}_{i \geq 1}$ is finite.

According to the induction assumption, $\left\{t_{k-2, k-1}^{(i)}\right\}_{i \geq 1}$ is finite and one has

$\forall t \geq t_{k-2, k-1}^{\left(i_{k-1}\right)},\left|\tilde{x}_{1}(t)\right| \leq \frac{v}{\gamma_{k} \theta^{k-1}}$.

Without loss of generality, assume that $i_{w} \leq k$. According to (34), the first $k$ equations of the observation error system related to observer (30) can be written under the following condensed form for all $t \geq t_{k-2, k-1}^{\left(i_{k-1}\right)}$,

$\underline{\tilde{\tilde{x}}}_{k}=A_{k} \underline{\tilde{x}}_{k}+\underline{\tilde{\varphi}}_{k}^{s}\left(u, \underline{\hat{x}}_{k}, \underline{x}_{k}\right)$

$$
\begin{aligned}
& -\theta \Delta_{k}^{-1}(\theta) \Gamma_{k} C_{k}^{T} \tilde{x}_{1}(t)+B_{k, k} \tilde{x}_{k+1}(t) \\
& -B_{k, i_{w}} w(t),
\end{aligned}
$$

where $\underline{\tilde{x}}_{k}, \underline{\hat{x}}_{k}, \underline{x}_{k}, \underline{\varphi}_{k}^{s} \in \mathbb{R}^{k}$ are the respective subvectors of $\tilde{x}, \hat{x}, x$ and $\varphi^{s}$ defined as in (7), $A_{k}$ as in (1), $\left(C_{k}\right)$ as in (2), $\Gamma_{k}$ as in (4), $\Delta_{k}$ as in (6), $B_{k, k}$ as in (3) with $k_{0}=k$ and $B_{k, i_{w}}$ as in (3) with $k_{0}=i_{w}$. Recall that $\bar{A}_{k}=A_{k}-\Gamma_{k} C_{k}$ is Hurwitz and therefore there exist a SPD $P_{k}$ and a positive real $\mu_{k}$ satisfying inequality (8).

Notice that the error system (99) has the same structure as the error system (25) and the term $\tilde{x}_{k+1}(t)$ in (99) plays the role of $\varepsilon(t)$ in (25). Moreover, one recalls that according to (37), one has

$\left|\tilde{x}_{j}(t)\right| \leq \alpha_{\theta}, j=1, \ldots, n$,

for any $t<T$ where $T>0$ is any fixed arbitrarily large positive number.

Besides, the essential bound $\delta_{w}$ is assumed to satisfy inequality (28). According to these facts, the observation error $\underline{\tilde{x}}_{j}, j=1, \ldots, k$, satisfies an inequality similar to (29) for all $t \geq t_{k-2, k-1}^{\left(i_{k-1}\right)}$. such inequality specializes as follows

$$
\begin{aligned}
\left|\tilde{x}_{j}(t)\right| \leq & \left(\theta^{k-1} e^{-\theta \beta_{k}\left(t-t_{k-2, k-1}^{\left(i_{k-1}\right)}\right)}\right. \\
& \left.+\frac{1}{\theta \beta_{k}}\right) \sigma_{P_{k}} \bar{\alpha}_{\theta}, \quad j=1, \ldots, k,
\end{aligned}
$$

where $\beta_{k}$ is defined as in (27) with $n$ substituted by $k$, $P_{k}=P_{k}^{T}$ and

$\bar{\alpha}_{\theta}=\alpha_{\theta}+\bar{\delta}_{w}$.

Let $t_{k-1}^{\star}>t_{k-2, k-1}^{\left(i_{k-1}\right)}$ be a time instant such that

$$
\begin{aligned}
& \theta^{k-1} e^{-\theta \beta_{k}\left(t_{k-1}^{\star}-t_{k-2, k-1}^{\left(i_{k-1}\right)}\right)}=\frac{1}{\theta \beta_{k}}, \\
& \text { i.e. } t_{k-1}^{\star}=t_{k-2, k-1}^{\left(i_{k-1}\right)}+\frac{1}{\theta \beta_{k}}\left(k \log (\theta)+\log \left(\beta_{k}\right)\right) .
\end{aligned}
$$

According to the definition of $t_{k-1}^{\star}$ and using (101), one has

$\forall t \geq t_{k-1}^{\star}, \quad\left|\tilde{x}_{j}(t)\right| \leq 2 \frac{\sigma_{P_{k}}}{\beta_{k}}\left(\bar{\alpha}_{\theta} / \theta\right), \quad j=1, \ldots, k$.

Notice that according to the induction assumption, $\left|\tilde{x}_{1}(t)\right|$ cannot come back to $\mathcal{S}_{k-2}$ for all $t \geq t_{k-2, k-1}^{\left(i_{k-1}\right)}$. Hence, according to Remark 3.2, $\forall t \geq t_{k-1}^{\star},\left|\tilde{x}_{1}(t)\right|$ will evolve throughout the sectors $\mathcal{S}_{k-1}, \ldots, \mathcal{S}_{n-1}$. 
Assume that there exists $t_{k, k-1}^{(i)}>t_{k-1}^{\star}$, where $i$ is a positive integer, at which $\left|\tilde{x}_{1}(t)\right|$ leaves $\mathcal{S}_{k}$ and enters $\mathcal{S}_{k-1}$ for the $i$ 'th time. Notice that if such a time instant does not exist then the induction is proved.

Set

$\eta_{k} \triangleq 6 \frac{\bar{L}_{\varphi} \sigma_{P_{k}}}{\beta_{k}}+1 \quad$ with $\quad \bar{L}_{\varphi}=\max \left(L_{\varphi}, 1\right)$.

One shall prove the following property which is needed in the remaining of the proof,

$$
\begin{aligned}
& \exists \bar{\theta}_{0}>0 ; \forall \theta>\bar{\theta}_{0} ; \quad \exists \bar{t}_{k, k-1}^{(i)}>t_{k, k-1}^{(i)} ; \\
& \forall t \geq \bar{t}_{k, k-1}^{(i)}, \frac{\eta_{k}}{\theta} \sum_{j=1}^{k} \frac{\left(t-t_{k, k-1}^{(i)}\right)^{j}}{j !} \\
& \leq \frac{1}{2} \frac{\left(t-t_{k, k-1}^{(i)}\right)^{k}}{k !}, \\
& \text { with } \lim _{\theta \rightarrow \infty}\left(\bar{t}_{k, k-1}^{(i)}-t_{k, k-1}^{(i)}\right)=0 .
\end{aligned}
$$

To prove property (106), set $\bar{\tau}=t-t_{k, k-1}^{(i)}$ and consider the following polynomial in $\bar{\tau}$

$$
\bar{P}(\bar{\tau})=\left(\frac{\eta_{k}}{\theta} \sum_{j=1}^{k} \frac{\bar{\tau}^{j-1}}{j !}\right)-\frac{1}{2} \frac{\bar{\tau}^{k-1}}{k !} .
$$

Using the Descartes' rule of signs, the equation $\bar{P}(\bar{\tau})=$ 0 admits a unique positive real root, say $\bar{\tau}_{k, k-1}^{(i)}$. Since $\bar{P}(0)=\frac{\eta_{k}}{\theta}>0$, one has: $\forall \bar{\tau}>\bar{\tau}_{k, k-1}^{(i)}, \bar{P}(\bar{\tau})<0$ which also implies that $\bar{\tau} \bar{P}(\bar{\tau}) \leq 0$. According to the definition of $\bar{\tau}$, the expression of the positive real $\bar{t}_{k, k-1}^{(i)}$ involved in (106) is $\bar{t}_{k, k-1}^{(i)}=t_{k, k-1}^{(i)}+\bar{\tau}_{k, k-1}^{(i)}$. Moreover, $\bar{\tau}_{k, k-1}^{(i)}$ satisfies

$\frac{\eta_{k}}{\theta} \sum_{j=1}^{k} \frac{\left(\bar{\tau}_{k, k-1}^{(i)}\right)^{j-1}}{j !}=\frac{1}{2} \frac{\left(\bar{\tau}_{k, k-1}^{(i)}\right)^{k-1}}{k !}$.

The left side of the above equality is a polynomial in $\bar{\tau}_{k, k-1}^{(i)}$ and all its coefficients tend to zero when when $\theta$ goes to infinity. This implies that $\lim _{\theta \rightarrow \infty} \bar{\tau}_{k, k-1}^{(i)}=0$ and the property (106) is hence proved.

Now, let $t>t_{k, k-1}^{(i)}$ such that $\left|\tilde{x}_{1}(t)\right| \in \mathcal{S}_{k-1}$. Then, one has [compare with (69)]

$$
\begin{aligned}
\left|\tilde{x}_{1}(t)\right| \leq & \sum_{j=0}^{n-1} \frac{\left(t-t_{k, k-1}^{(i)}\right)^{j}}{j !}\left|\tilde{x}_{j+1}\left(t_{k, k-1}^{(i)}\right)\right| \\
& +\sum_{j=1}^{n} \int_{t_{k, k-1}^{(i)}}^{t} \frac{(t-s)^{j-1}}{(j-1) !}\left|\tilde{\varphi}_{j}^{s}(u, \hat{x}, x)\right| \mathrm{d} s
\end{aligned}
$$

$$
\begin{aligned}
& +\delta_{\varepsilon} \frac{\left(t-t_{k, k-1}^{(i)}\right)^{n}}{n !}+\delta_{w} \frac{\left(t-t_{k, k-1}^{(i)}\right)^{i_{w}}}{i_{w} !} \\
& -\theta v \sum_{j=k+1}^{n} \frac{\left(t-t_{k, k-1}^{(i)}\right)^{j}}{j !}
\end{aligned}
$$

Since $\delta_{w}$ satisfies (28) and from the fact that $n-i_{w} \geq$ 1 , the above inequality leads to

$$
\begin{aligned}
\left|\tilde{x}_{1}(t)\right| \leq & \sum_{j=0}^{n-1} \frac{\left(t-t_{k, k-1}^{(i)}\right)^{j}}{j !}\left|\tilde{x}_{j+1}\left(t_{k, k-1}^{(i)}\right)\right| \\
& +\sum_{j=1}^{n} \int_{t_{k, k-1}^{(i)}}^{t} \frac{(t-s)^{j-1}}{(j-1) !}\left|\tilde{\varphi}_{j}^{s}(u, \hat{x}, x)\right| d s \\
& +\delta_{\varepsilon} \frac{\left(t-t_{k, k-1}^{(i)}\right)^{n}}{n !}+\frac{\bar{\delta}_{w}}{\theta} \frac{\left(t-t_{k, k-1}^{(i)}\right)^{i_{w}}}{i_{w} !} \\
& -\theta v \sum_{j=k+1}^{n} \frac{\left(t-t_{k, k-1}^{(i)}\right)^{j}}{j !} \\
& \triangleq \underline{Q}(t)+\bar{Q}(t),
\end{aligned}
$$

with

$$
\left\{\begin{aligned}
\underline{Q}(t)= & \sum_{j=0}^{k-1} \frac{\left(t-t_{k, k-1}^{(i)}\right)^{j}}{j !}\left|\tilde{x}_{j+1}\left(t_{k, k-1}^{(i)}\right)\right| \\
& +\sum_{j=1}^{k} \int_{t_{k, k-1}^{(i)}}^{t} \frac{(t-s)^{j-1}}{(j-1) !}\left|\tilde{\varphi}_{j}^{s}(u, \hat{x}, x)\right| \mathrm{d} s \\
& +\frac{\bar{\delta}_{w}}{\theta} \frac{\left(t-t_{k, k-1}^{(i)}\right)^{i w}}{i_{w} !} \\
\bar{Q}(t)= & \sum_{j=k}^{n-1} \frac{\left(t-t_{k, k-1}\right)^{j}}{j !}\left|\tilde{x}_{j+1}\left(t_{k, k-1}^{(i)}\right)\right| \\
& +\sum_{j=k+1}^{n} \int_{t_{k, k-1}^{t}}^{t} \frac{(t-s)^{j-1}}{(j-1) !}\left|\tilde{\varphi}_{j}^{s}(u, \hat{x}, x)\right| \mathrm{d} s \\
& +\delta_{\varepsilon} \frac{\left(t-t_{k, k-1}^{(i)}\right)^{n}}{n !}-\theta v \sum_{j=k+1}^{n} \frac{\left(t-t_{k, k-1}^{(i)}\right)^{j}}{j !} .
\end{aligned}\right.
$$

According to (18) and (37), one has for $j=k+$ $1, \ldots, n$,

$\left|\tilde{\varphi}_{j}^{s}(u, \hat{x}, x)\right| \leq 2 n L_{\varphi} R \quad$ and $\quad\left|\tilde{x}_{j}\left(t_{k, k-1}^{(i)}\right)\right| \leq \alpha_{\theta} \cdot(110)$

Using (104) and (110), $\bar{Q}(t)$ can be bounded as follows

$$
\begin{aligned}
\bar{Q}(t) \leq & \bar{\alpha}_{\theta} \frac{\left(t-t_{k, k-1}^{(i)}\right)^{k}}{k !}+\alpha_{\theta} \sum_{j=k+1}^{n-1} \frac{\left(t-t_{k, k-1}^{(i)}\right)^{j}}{j !} \\
& +2 n L_{\varphi} R \sum_{j=k+1}^{n} \frac{\left(t-t_{k, k-1}^{(i)}\right)^{j}}{j !} \\
& +\delta_{\varepsilon} \frac{\left(t-t_{k, k-1}^{(i)}\right)^{n}}{n !}-\theta v \sum_{j=k+1}^{n} \frac{\left(t-t_{k, k-1}^{(i)}\right)^{j}}{j !} \\
\leq & \bar{\alpha}_{\theta} \frac{\left(t-t_{k, k-1}^{(i)}\right)^{k}}{k !}+\left(\delta_{\theta}+2 n L_{\varphi} R-\theta v\right)
\end{aligned}
$$




$$
\sum_{j=k+1}^{n} \frac{\left(t-t_{k, k-1}^{(i)}\right)^{j}}{j !},
$$

where $\bar{\alpha}_{\theta}$ is as given by (102), $\delta_{\theta}=\max \left(\alpha_{\theta}, \delta_{\varepsilon}\right)$. Note that since $\lim _{\theta \rightarrow \infty}\left(\bar{\alpha}_{\theta} / \theta\right)=0$, one has $\lim _{\theta \rightarrow \infty}\left(\delta_{\theta} / \theta\right)$ $=0$.

Now, choose $\theta$ high enough such that $\delta_{\theta}+2 n L_{\varphi} R-$ $\nu \theta \leq-\frac{v \theta}{2}$. Note that such a choice is possible since $\lim _{\theta \rightarrow \infty}\left(\delta_{\theta} / \theta\right)=0$. With such a choice, inequality (111) becomes

$$
\begin{aligned}
\bar{Q}(t) & \leq \bar{\alpha}_{\theta} \frac{\left(t-t_{k, k-1}^{(i)}\right)^{k}}{k !}-\frac{\nu \theta}{2} \sum_{j=k+1}^{n} \frac{\left(t-t_{k, k-1}^{(i)}\right)^{j}}{j !} \\
& \leq \bar{\alpha}_{\theta} \frac{\left(t-t_{k, k-1}^{(i)}\right)^{k}}{k !}-\frac{\nu \theta}{2} \frac{\left(t-t_{k, k-1}^{(i)}\right)^{k+1}}{(k+1) !} .
\end{aligned}
$$

Let us now bound $\underline{Q}(t)$. Indeed, according to (104) and since $t_{k, k-1}^{(i)}>t_{k-1}^{\star}$, one has for $j=1, \ldots, k-1$, $\left|\tilde{x}_{j+1}\left(t_{k, k-1}^{(i)}\right)\right| \leq 2 \frac{\sigma_{P_{k}}}{\beta_{k}}\left(\bar{\alpha}_{\theta} / \theta\right)$.

Similarly, $\forall s \in\left[t_{k, k-1}^{(i)}, t\right]$, one has for $j=1, \ldots, k$, $\left|\tilde{\varphi}_{j}^{s}(u, \hat{x}, x)\right| \leq 2 L_{\varphi}\left|\tilde{x}_{j}\right| \leq 4 \frac{L_{\varphi} \sigma_{P_{k}}}{\beta_{k}}\left(\bar{\alpha}_{\theta} / \theta\right)$.

Using (113) and (114) and from the fact that $1 \leq$ $i_{w} \leq k, \underline{Q}(t)$ defined by (109) can be bounded as follows

$$
\begin{aligned}
\underline{Q}(t) \leq & \left|\tilde{x}_{1}\left(t_{k, k-1}^{(i)}\right)\right|+2 \frac{\sigma_{P_{k}}}{\beta_{k}}\left(\bar{\alpha}_{\theta} / \theta\right) \sum_{j=1}^{k-1} \frac{\left(t-t_{k, k-1}^{(i)}\right)^{j}}{j !} \\
& +\left(4 \frac{L_{\varphi} \sigma_{P_{k}}}{\beta_{k}}\left(\bar{\alpha}_{\theta} / \theta\right)+\frac{\bar{\delta}_{w}}{\theta}\right) \sum_{j=1}^{k} \frac{\left(t-t_{k, k-1}^{(i)}\right)^{j}}{j !} \\
\leq & \left|\tilde{x}_{1}\left(t_{k, k-1}^{(i)}\right)\right|+\left(6 \frac{\bar{L}_{\varphi} \sigma_{P_{k}}}{\beta_{k}}+1\right)\left(\frac{\bar{\alpha}_{\theta}}{\theta}\right) \\
& \sum_{j=1}^{k} \frac{\left(t-t_{k, k-1}^{(i)}\right)^{j}}{j !} \text { since } \bar{\delta}_{w} \leq \bar{\alpha}_{\theta} \\
= & \left|\tilde{x}_{1}\left(t_{k, k-1}^{(i)}\right)\right|+\left(\frac{\eta_{k}}{\theta} \sum_{j=1}^{k} \frac{\left(t-t_{k, k-1}^{(i)}\right)^{j}}{j !}\right) \bar{\alpha}_{\theta},
\end{aligned}
$$

where $\eta_{k}$ and $\bar{L}_{\varphi}$ are given by (105).

Hence, for $t \geq \bar{t}_{k, k-1}^{(i)}$ and using property (106), inequality (115) leads to

$\underline{Q}(t) \leq\left|\tilde{x}_{1}\left(t_{k, k-1}^{(i)}\right)\right|+\frac{\bar{\alpha}_{\theta}}{2} \frac{\left(t-t_{k, k-1}^{(i)}\right)^{k}}{k !}$.
Combining (108), (112) and (116), one gets for all $t \geq \bar{t}_{k-1, k}^{(i)}$,

$$
\begin{aligned}
\left|\tilde{x}_{1}(t)\right| \leq & \left|\tilde{x}_{1}\left(t_{k, k-1}^{(i)}\right)\right|+\frac{3}{2} \bar{\alpha}_{\theta} \frac{\left(t-t_{k, k-1}^{(i)}\right)^{k}}{k !} \\
& -\frac{\nu \theta}{2} \frac{\left(t-t_{k, k-1}^{(i)}\right)^{k+1}}{(k+1) !} \\
\leq & \left|\tilde{x}_{1}\left(t_{k, k-1}^{(i)}\right)\right|+\frac{3}{2} \bar{\alpha}_{\theta} \frac{\left(t-t_{k, k-1}^{(i)}\right)^{k}}{k !} \\
& -\frac{\nu \theta}{2} \frac{\left(t-t_{k, k-1}^{(i)}\right)^{k}\left(t-\bar{t}_{k, k-1}^{(i)}\right)}{(k+1) !} \\
= & \frac{v}{\gamma_{k+1} \theta^{k}}+\frac{\nu \theta}{2(k+1) !}\left(t-t_{k, k-1}^{(i)}\right)^{k} \\
& \left(\frac{3(k+1) \bar{\alpha}_{\theta}}{\nu \theta}-\left(t-\bar{t}_{k, k-1}^{(i)}\right)\right),
\end{aligned}
$$

Now, set

$t_{k, k-1}^{\star}=\bar{t}_{k, k-1}^{(i)}+\frac{3(k+1) \bar{\alpha}_{\theta}}{\nu \theta}$.

Combining (117) and (118), one gets for all $t \geq$ $t_{k, k-1}^{\star},\left|\tilde{x}_{1}(t)\right| \leq \frac{v}{\gamma_{k+1} \theta^{k}}$, i.e. $t_{k-1, k}^{\left(i_{k}\right)}=t_{k, k-1}^{\star}$. This ends the proof of Proposition 3.5.

\section{References}

1. Gauthier, J.P., Hammouri, H., Othman, S.: A simple observer for nonlinear systems - application to bioreactors. IEEE Trans. Auto. Control 37(6), 875-880 (1992)

2. Alessandri, A., Rossi, A.: Increasing-gain observers for nonlinear systems: stability and design. Automatica 57, 180 188 (2015)

3. Ciccarella, G., Dalla Mora, M., Germani, A.: A Luenbergerlike observer for nonlinear systems. Automatica 47, 537556 (1993)

4. Fliess, M., Join, C., Sira-Ramirez, H.: Nonlinear estimation is easy. Int. J. Model. Ident. Control 4, 12-27 (2008)

5. Gauthier, J.P., Kupka, I.: Deterministic Observation Theory and Applications. Cambridge University Press, UK (2001)

6. Hammouri, H., Bornard, G., Busawon, K.: High gain observer for structured multi-output nonlinear systems. IEEE Trans. Autom. Control 55, 987-992 (2010)

7. Ren, C., Tong, S., Li, Y.: Fuzzy adaptive high-gain-based observer backstepping control for SISO nonlinear systems with dynamical uncertainties. Nonlinear Dyn. 67, 941-955 (2012)

8. Khalil, H.H., Praly, L.: High-gain observers in nonlinear feedback control. Int. J. Robust Nonlinear Control 48, 9931015 (2014)

9. Yu, X., Yang, J., Li, S.: Disturbance observer-based autonomous landing control of unmanned helicopters on moving shipboard. Nonlinear Dyn. 102, 131-150 (2020) 
10. Du, M., Zhao, D., Yang, M., Chen, H.: Nonlinear extended state observer-based output feedback stabilization control for uncertain nonlinear half-car active suspension systems. Nonlinear Dyn. 100, 2483-2503 (2020)

11. Khalil, H.H.: High-gain observers in nonlinear feedback control. SIAM, Philadelphia (2017)

12. Astolfi, D., Zaccarian, L., Jungers, M.: On the use of lowpass filters in high-gain observers. Syst. Control Lett. 148, 104856 (2021)

13. Astolfi, D., Alessandri, A., Zaccarian, L.: Stubborn and dead-zone redesign for nonlinear observers and filters. IEEE Trans. Auto. Control 66(2), 667-682 (2020)

14. Tréangle, C., Farza, M., M'Saad, M.: Filtered high gain observer for a class of uncertain nonlinear systems with sampled outputs. Automatica 101, 197-206 (2019)

15. Astolfi, D., Marconi, L.: A high-gain nonlinear observer with limited gain power. IEEE Trans. Autom. Control 60, 30593064 (2015)

16. Oueder, M., Farza, M., Ben Abdennour, R., M'Saad, M.: A high gain observer with updated gain for a class of mimo non triangular systems. Syst. Control Lett. 61, 298-308 (2012)

17. Boizot, N., Busvelle, E., Gauthier, J.P.: An adaptive highgain observer for nonlinear systems. Automatica 46, 14831488 (2010)

18. Teel, A.: Further variants of the Astolfi/Marconi high gain observer. In: Proc. of the American Control Conference, Boston, pp. 993-998 (2016)

19. Khalil, H.H.: Cascade high-gain observers in output feedback control. Automatica 80, 110-118 (2017)

20. Astolfi, D., Marconi, L., Praly, L., Teel, A.: Low-power peaking-free high-gain observers. Automatica 98, 169-179 (2018)

21. Astolfi, D., Bernard, P., Postoyan, R., Marconi, M.: Constrained state estimation for nonlinear systems: a redesign approach based on convexity. IEEE Trans. Auto. Control. (2021). https://doi.org/10.1109/TAC.2021.3064537
22. Heemels, W.P.M.H., Camlibel, M.K., Heertjes, M.F.: Oblique projected dynamical systems and incremental stability under state constraints. IEEE Control Syst. Lett. 44(4), 1060-1065 (2020)

23. Bouraoui, I., Farza, M., Menard, T., Ben Abdennour, R., M'Saad, M., Mosrati, H.: Observer design for a class of uncertain nonlinear systems with sampled outputsapplication to the estimation of kinetic rates in bioreactors. Automatica 55, 78-87 (2015)

24. Dimitrov, D.K., Peña, J.M.: Almost strict total positivity and a class of Hurwitz polynomials. J. Approx. Theory 132, 212$223(2005)$

25. Ahrens, J.H., Khalil, H.: High-gain observer in the presence of measurement noise: a switched approach. Automatica 45, 936-943 (2009)

26. Busawon, K.K., Kabore, P.: Disturbance attenuation using proportional integral observers. Int. J. Control 74, 618-627 (2001)

27. Ibrir, S.: Robust state estimation with q-integral observers. In: Proc. of the American Control Conference, Boston, pp. 3466-3471 (2016)

28. Wang, L., Astolfi, D., Marconi, L., Su, H.: High-gain observers with limited gain power for systems with observability canonical form. Automatica 75, 16-23 (2017)

29. Albert, A.A.: An inductive proof of Descartes' rule of signs. Am. Math. Month. 50, 178-180 (1943)

Publisher's Note Springer Nature remains neutral with regard to jurisdictional claims in published maps and institutional affiliations. 